\title{
Solution of Vizings Problem on Interchanges for the case of Graphs with Maximum Degree 4 and Related Results
}

\author{
Armen Asratian and Carl Johan Casselgren
}

Journal Article

\section{Tweet}

N.B.: When citing this work, cite the original article.

Original Publication:

Armen Asratian and Carl Johan Casselgren, Solution of Vizings Problem on Interchanges for the case of Graphs with Maximum Degree 4 and Related Results, Journal of Graph Theory, 2016. 82(4), pp.350-373.

http://dx.doi.org/10.1002/jgt.21906

Copyright: Wiley: 12 months

http://eu.wiley.com/WileyCDA/

Postprint available at: Linköping University Electronic Press

http://urn.kb.se/resolve?urn=urn:nbn:se:liu:diva-130653

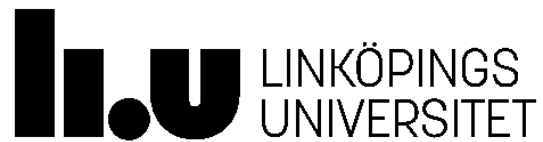




\title{
Solution of Vizing's Problem on Interchanges for the case of Graphs with Maximum Degree 4 and Related Results
}

\author{
Armen S. Asratian* \\ Department of Mathematics \\ Linköping University \\ SE-581 83 Linköping, Sweden
}

\author{
Carl Johan Casselgren ${ }^{\dagger}$ \\ Department of Mathematics \\ Linköping University \\ SE-581 83 Linköping, Sweden
}

August 17, 2015

\begin{abstract}
Let $G$ be a Class 1 graph with maximum degree 4 and let $t \geq 5$ be an integer. We show that any proper $t$-edge coloring of $G$ can be transformed to any proper 4-edge coloring of $G$ using only transformations on 2-colored subgraphs (so-called interchanges). This settles the smallest previously unsolved case of a well-known problem of Vizing on interchanges, posed in 1965. Using our result we give an affirmative answer to a question of Mohar for two classes of graphs: we show that all proper 5-edge colorings of a Class 1 graph with maximum degree 4 are Kempe equivalent, that is, can be transformed to each other by interchanges, and that all proper 7-edge colorings of a Class 2 graph with maximum degree 5 are Kempe equivalent.
\end{abstract}

\section{Introduction}

We consider finite graphs without loops and multiple edges. A proper $t$-edge coloring of a graph $G$ is a mapping $f: E(G) \longrightarrow\{1, \ldots, t\}$ such that $f(e) \neq f\left(e^{\prime}\right)$ for every pair of adjacent edges $e$ and $e^{\prime}$ in $G$. If $e \in E(G)$ and $f(e)=k$ then we say that the edge $e$ is colored $k$ under $f$. The set of edges colored $k$ under $f$ we denote by $M(f, k)$. We denote by $\Delta(G)$ the maximum degree of vertices of a graph $G$, and by $d_{G}(v)$ the degree of a vertex $v$ of $G$. The chromatic index $\chi^{\prime}(G)$ of a graph $G$ is the minimum number $k$ for which there exists a proper $k$-edge coloring of $G$. For a proper $t$-edge coloring $f$ of $G$ and any two colors $a, b \in\{1, \ldots, t\}$ we denote by $G_{f}(a, b)$ the subgraph induced by the set $M(f, a) \cup M(f, b)$. By switching colors $a$ and $b$ on a connected component of $G_{f}(a, b)$, we obtain another proper $t$-edge coloring of $G$. This operation is called an interchange or a Kempe change. In the following, if a $t$-edge coloring is transformed to a $t^{\prime}$-edge coloring by a sequence of interchanges and $t^{\prime} \leq t$, then

\footnotetext{
*E-mail address: armen.asratian@liu.se

${ }^{\dagger}$ E-mail address: carl.johan.casselgren@liu.se Part of the work done while the author was a postdoc at University of Southern Denmark. Research supported by SVeFUM.
} 
we only allow colors $1,2, \ldots, t$ to be involved in the interchanges. Two $t$-edge colorings $f, g$ are Kempe equivalent if $g$ can be transformed to $f$ by a sequence of interchanges.

Interchanges play a key role in investigations on edge colorings. The proofs of many results in this area are based on transformations of one proper edge coloring of a graph $G$ to another using interchanges. For example, Vizing's theorem on the chromatic index [9] can be reformulated, taking into consideration the proof of it, in the following way.

Theorem A. For every graph $G, \chi^{\prime}(G) \leq \Delta(G)+1$. Moreover every proper $t$-edge coloring of $G$, where $t \geq \Delta(G)+2$, can be transformed to a proper $(\Delta(G)+1)$-edge coloring of $G$ by a sequence of interchanges.

Vizing's result implies that for any graph $G, \chi^{\prime}(G)=\Delta(G)$ or $\chi^{\prime}(G)=\Delta(G)+1$. In the former case $G$ is said to be Class 1, and in the latter $G$ is Class 2. For a Class 2 graph $G$ the result of Vizing means that any proper edge coloring of $G$ can be transformed to a proper $\chi^{\prime}(G)$-edge coloring by using interchanges only. The following problem posed by Vizing [10, 11] (see also [8, 5]) for Class 1 graphs is still open:

Problem 1. Is it true that any Class 1 graph $G$ satisfies the following property: every proper t-edge coloring of $G, t \geq \Delta(G)+1$, can be transformed to a proper $\Delta(G)$-edge coloring of $G$ by a sequence of interchanges? (This property we shall call the Vizing property.)

Mohar [7] used the edge coloring algorithm suggested by Vizing [10] to prove that for a graph $G$ with a proper $t$-edge coloring $f$, every proper $\chi^{\prime}(G)$-edge coloring can be obtained from $f$ by a sequence of interchanges, provided that $t \geq \chi^{\prime}(G)+2$, i.e. he solved Problem 1 for the case when $t \geq \Delta(G)+2$. This means that all proper $t$-edge colorings of $G$ are Kempe equivalent if $t \geq \chi^{\prime}(G)+2$. Mohar also posed the following:

Problem 2. Is it true that all proper $\left(\chi^{\prime}(G)+1\right)$-edge colorings of a graph $G$ are Kempe equivalent?

For graphs $G$ with $\Delta(G) \leq 3$, Problem 2 was resolved to the positive by McDonald et al. [6]. In particular they proved the following:

Theorem B [6]. Let $G$ be a graph with $\Delta(G) \leq 3$. Then all proper $(\Delta(G)+1)$-edge colorings of $G$ are Kempe equivalent.

In the general case the situation is different. It is shown in [6] that if $\chi^{\prime}(G)$ is replaced by $\Delta(G)$ in Problem 2 , then we get a problem which in general has a negative answer.

Note that it follows from Theorems A and B that all 5-edge colorings of a graph with maximum degree 3 are Kempe equivalent; so Theorem B combined with Theorem A settles Problem 2 for graphs with maximum degree 3. McDonald et al. [6] obtained also an affirmative answer to Problem 2 for some Class 2 graphs:

Theorem C [6]. Let $G$ be a graph with $\Delta(G)=4$ and $\chi^{\prime}(G)=5$. Then all proper 6-edge colorings of $G$ are Kempe equivalent.

Our first result establishes a connection between Problem 1 and Problem 2. 
Theorem 1.1. Let $k \geq 2$ be an integer. Then all Class 1 graphs with maximum degree at most $k$ have the Vizing property if and only if for every graph $G$ with $\Delta(G) \leq k$ all proper $\left(\chi^{\prime}(G)+1\right)$-edge colorings are Kempe equivalent.

Our second result establishes a connection between the solution of Problem 2 for graphs with maximum degree $k-1$ and Class 2 graphs with maximum degree $k$.

Theorem 1.2. Let $k \geq 4$ be an integer. If for every graph $H$ with $\Delta(H)=k-1$ all proper $\left(\chi^{\prime}(H)+1\right)$-edge colorings are Kempe equivalent, then for every Class 2 graph $G$ with maximum degree $k$ all proper $(k+2)$-edge colorings are Kempe equivalent. B.

In the case $k=4$, Theorem 1.2 actually implies that Theorem $\mathrm{C}$ follows from Theorem

The main result of this paper is the following theorem which gives a positive answer to the smallest previously unsolved case of Problem 1.

Theorem 1.3. Let $G$ be a Class 1 graph with $\Delta(G)=4$ and $t$ be an integer, $t \geq 5$. Then every proper $t$-edge coloring of $G$ can be transformed to any proper 4-edge coloring of $G$ by a sequence of interchanges.

By Theorem 1.3, any two proper 5-edge colorings of a Class 1 graph $G$ with $\Delta(G)=4$ can be transformed by interchanges to the same proper 4-edge coloring of $G$. This implies the next result, which gives a positive answer to the smallest previously unsolved case of Problem 2.

Corollary 1.4. If $G$ is a Class 1 graph with $\Delta(G)=4$, then all proper 5 -edge colorings of $G$ are Kempe equivalent.

Theorem 1.2, Theorem C and Corollary 1.4 imply the following result:

Corollary 1.5. Let $G$ be a Class 2 graph with $\Delta(G)=5$. Then all proper 7-edge colorings of $G$ are Kempe equivalent.

Note that Corollary 1.4, Theorem B, and Theorem 1.2 imply that Problem 2 has an affirmative answer for all graphs with maximum degree at most 4. Additionally, Corollary 1.5 settles the question for Class 2 graphs with maximum degree 5, so the smallest unsolved case is Class 1 graphs of maximum degree 5 .

Next, we describe some Class 1 graphs $G$ with $\Delta(G) \geq 5$ for which all proper $\left(\chi^{\prime}(G)+1\right)$ edge colorings are Kempe equivalent. Denote by $G_{\geq 5}$ the subgraph of $G$ induced by the set of vertices with degree at least 5 .

Theorem 1.6. For every graph $G$ with $\Delta(G) \geq 5$ where the subgraph $G_{\geq 5}$ is acyclic, all proper $\left(\chi^{\prime}(G)+1\right)$-edge colorings of $G$ are Kempe equivalent.

By Corollary 1.4, all proper 5-edge colorings of a Class 1 graph with maximum degree 4 are Kempe equivalent. Let us now briefly consider the problem of transforming a proper 4-edge coloring of such a graph $G$ to another proper 4-edge coloring of $G$. The next example shows that there are such graphs with two proper 4-edge colorings $f$ and $g$ such that $f$ cannot 

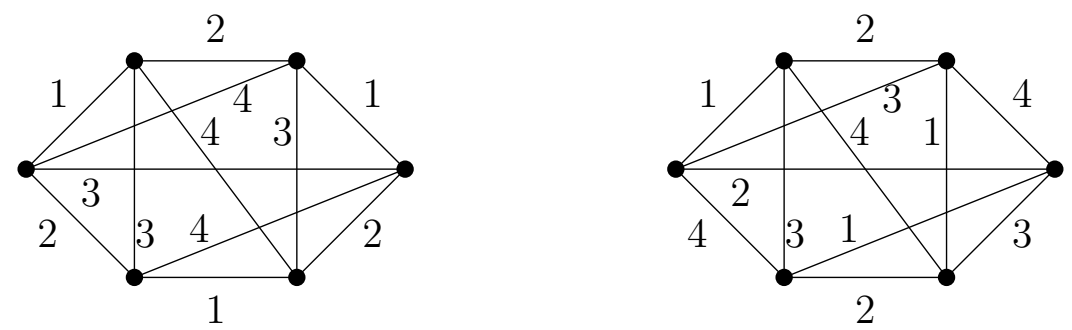

Figure 1: A graph with the edge colorings $f$ (to the left) and $g$ (to the right).

be transformed to $g$ even if, in addition to interchanges, one is allowed to use transformations on 3-edge colored subgraphs.

Consider the graph $G$ in Figure 1 with two different proper 4-edge colorings $f$ (to the left) and $g$ (to the right). Clearly, $g$ cannot be obtained from $f$ by renaming the colors. On the other hand any proper 3 -edge coloring of a subgraph $G\left(t_{1}, t_{2}, t_{3}\right)$ induced by the set of edges $M\left(f, t_{1}\right) \cup M\left(f, t_{2}\right) \cup M\left(f, t_{3}\right)$ gives the same partition of edges of $G\left(t_{1}, t_{2}, t_{3}\right)$ into perfect matchings, for any $1 \leq t_{1}<t_{2}<t_{3} \leq 5$. This follows from the fact that $G\left(t_{1}, t_{2}, t_{3}\right)$ contains a triangle and edges of this triangle belong to disjoint, uniquely defined perfect matchings. Therefore $g$ cannot be obtained from $f$ even by transformations on 2-edge colored and 3-edge colored subgraphs.

For a bipartite graph $G$ with $\Delta(G)=4$, any two proper 4-edge colorings can always be transformed into each other by recoloring 2-edge colored and 3-edge colored subgraphs. This follows from the result of Asratian and Mirumian [2] (see also [1]) that if $G$ is a bipartite graph with $\Delta(G) \geq 4$, then any proper $\Delta(G)$-edge coloring of $G$ can be transformed to any other proper $\Delta(G)$-edge coloring of $G$ in such a way that each intermediate coloring is a proper $\Delta(G)$-edge coloring and differs from the previous coloring by a 2- or 3-edge colored subgraph.

\section{Proofs of Theorem 1.1 and Theorem 1.2}

In this section we prove Theorem 1.1 and Theorem 1.2 .

First we introduce some terminology and notation. Let $\varphi$ be a proper $t$-edge coloring of $G$. For a vertex $v \in V(G)$, we say that a color $i$ appears at $v$ under $\varphi$ if there is an edge $e$ incident to $v$ with $\varphi(e)=i$, and we set

$$
\varphi(v)=\{\varphi(e): e \in E(G) \text { and } e \text { is incident to } v\} .
$$

If $\varphi$ is a proper $t$-edge coloring of $G$ and $1 \leq a, b \leq t$, then a path or cycle in $G_{\varphi}(a, b)$ is called $(a, b)$-colored under $\varphi$. We also say that such a path or cycle is bicolored under $\varphi$. A $\varphi$-fan at a vertex $v \in V(G)$ is a sequence $\left(e_{1}, e_{2}, \ldots, e_{n}\right)$ of edges such that

(i) the edges $e_{1}, e_{2}, \ldots, e_{n}$ are distinct and incident to $v$, 
(ii) if $e_{i}=v u_{i}$, then $\varphi\left(e_{i}\right)$ does not appear at $u_{i-1}, i=2, \ldots, n$.

For such a $\varphi$-fan, we say that color $\varphi\left(e_{i}\right)$ is associated with $u_{i-1}$. A $\varphi$-fan $\left(e_{1}, e_{2}, \ldots, e_{k}\right)$ at $v$ with $e_{k}=v u_{k}$ is saturated if there is a color $c$ such that $c \notin \varphi(v) \cup \varphi\left(u_{k}\right)$. For such a saturated $\varphi$-fan $\left(e_{1}, e_{2}, \ldots, e_{k}\right)$ we define a downshift as the following operation: define a new coloring $\varphi^{\prime}$ from $\varphi$ by setting $\varphi^{\prime}\left(e_{k}\right)=c, \varphi^{\prime}\left(e_{j}\right)=\varphi\left(e_{j+1}\right), j=1, \ldots, k-1$, and retaining the color of every other edge of $G$. Note that a downshift produces a new proper edge coloring and that it may be seen as a sequence of interchanges.

In all the above definitions, we often leave out the explicit reference to a coloring $\varphi$, if the coloring is clear from the context.

Now we present a family of graphs permitting an affirmative answer to Problem 1.

Denote by $G_{\Delta}$ the graph induced by the vertices of maximum degree in a graph $G$. It is known that if $G_{\Delta}$ is acyclic, then $G$ is a Class 1 graph [4]. In fact, Fournier [4] (see also [3]) described an algorithm for sequentially coloring the edges properly of such a graph using $\Delta(G)$ colors. This result also follows from Vizing's well-known adjacency lemma, see e.g. [5], p. 196. Here, we use a similar algorithm to prove the following proposition:

Proposition 2.1. Let $G$ be a graph where $G_{\Delta}$ is acyclic. Then any proper $t$-edge coloring of $G$ with $t>\Delta(G)$ can be transformed into a proper $\Delta(G)$-edge coloring by a sequence of interchanges.

Proof. The major parts of the proof is similar to the proof of Vizing's theorem on the chromatic intex. Let $G$ be a graph with maximum degree $\Delta$ such that $G_{\Delta}$ is acyclic, $t$ an integer satisfying $t>\Delta$, and suppose that $f$ is a proper $t$-edge coloring of $G$.

Taking Theorem A into consideration, it is sufficient to prove the proposition in the case $t=\Delta+1$.

Suppose that there is at least one edge $e$ colored $\Delta+1$ in $G$. We will show that using only interchanges we can transform $f$ to another proper $(\Delta+1)$-edge coloring with fewer edges colored $\Delta+1$.

Case A. There is an edge $e=v u_{1} \in E\left(G_{\Delta}\right)$ with $f(e)=\Delta+1$ and $d_{G_{\Delta}}(v)=1$.

Since every neighbor $x \neq u_{1}$ of $v$ has degree at most $\Delta-1$, there is a maximal fan $F=$ $\left(e_{1}, e_{2}, \ldots, e_{k}\right)$ at $v$ with $e_{i}=v u_{i}$ and with no vertex in $\left\{u_{1}, \ldots, u_{k}\right\}$ being associated with color $\Delta+1$. Suppose that $f\left(e_{i}\right)=c_{i}, i=1, \ldots, k$, where $c_{1}=\Delta+1$, and that color $c_{k+1} \neq \Delta+1$ does not appear at $u_{k}$.

Since $F$ is maximal, either

(i) there is no edge incident to $v$ colored $c_{k+1}$, or

(ii) $c_{k+1}=f\left(e_{j+1}\right)$ for some $j, 1 \leq j<k-1$, that is, $c_{k+1}=c_{j+1}$ and $c_{k+1}$ does not appear at $u_{j}$.

In the case (i) we downshift on $F$, and since no vertex in $\left\{u_{1}, \ldots, u_{k}\right\}$ is associated with color $\Delta+1$, we obtain a proper $(\Delta+1)$-edge coloring with fewer edges colored $\Delta+1$.

Suppose now that (ii) holds. Choose a color $c_{0}$ that does not appear at $v$. If $c_{0}$ does not appear also at $u_{k}$, then we replace $c_{k+1}$ with $c_{0}$ and proceed as in the case (i). Otherwise, we consider a maximal $\left(c_{0}, c_{k+1}\right)$-colored path $Q$ with origin at $u_{k}$. 
If $u_{j+1}$ is on $Q$, then $v$ is an endpoint of $Q$. We interchange the colors on $Q$ and denote the obtained coloring by $f_{1}$. The color $c_{k+1}$ does not appear now at vertices $v$ and $u_{j}$, so we may downshift on the saturated $f_{1}$-fan $\left(e_{1}, e_{2}, \ldots, e_{j}\right)$ to obtain the desired result.

If $u_{j}$ is on $Q$, then this is an endpoint of $Q$. We interchange the colors on $Q$ and obtain a coloring $f_{1}$ where the color $c_{0}$ does not appear at vertices $v$ and $u_{j}$. Now we downshift on the saturated $f_{1}$-fan $\left(e_{1}, \ldots, e_{j}\right)$.

If $\left\{u_{j-1}, u_{j}\right\} \cap V(Q)=\emptyset$, then we interchange colors on $Q$ and then downshift on $\left(e_{1}, \ldots, e_{k}\right)$.

Case B. There is no edge $e=u v \in E\left(G_{\Delta}\right)$ of color $\Delta+1$ with $d_{G_{\Delta}}(u)=1$ or $d_{G_{\Delta}}(v)=1$.

The following cases are possible:

Case B.1 $E\left(G_{\Delta}\right) \cap M(f, \Delta+1) \neq \emptyset$.

Let $e=u v \in E\left(G_{\Delta}\right) \cap M(f, \Delta+1)$. We will describe an algorithm for constructing a sequence of distinct vertices $a_{0}, a_{1}, \ldots, a_{n}$ in $G$ such that $a_{i} \in V\left(G_{\Delta}\right), i=0, \ldots, n$, and $a_{i}$ and $a_{i+1}$ are adjacent in $G$. The algorithm will also construct a related sequence $g_{1}, \ldots g_{n-1}$ of proper $(\Delta+1)$-edge colorings of $G$, such that the edge $a_{i} a_{i+1}$ is colored $\Delta+1$ under $g_{i}$. Additionally $\left|M\left(g_{i}, \Delta+1\right)\right| \leq|M(f, \Delta+1)|$ for each $i \in\{1, \ldots, n-1\}$.

\section{Algorithm}

Step 1: We first set $g_{1}=f$ and $a_{0}=u$ and $a_{1}=v$.

Step $i(i \geq 2)$ : Suppose that we have constructed the sequence $a_{0}, a_{1}, \ldots, a_{i-1}$ of distinct vertices in $G$, such that $a_{j-1}$ and $a_{j}$ are adjacent, $j=1, \ldots, i-1$, and a sequence of proper $(\Delta+1)$-edge colorings $g_{1}, \ldots g_{i-1}$, satisfying $\left|M\left(g_{j}, \Delta+1\right)\right| \leq|M(f, \Delta+1)|$ and $g_{j}\left(a_{j-1} a_{j}\right)=\Delta+1, j=1, \ldots, i-1$.

If $d_{G_{\Delta}}\left(a_{i-1}\right)=1$, then Stop. The coloring $g_{i-1}$ satisfies the conditions in Case A and we can transform $g_{i-1}$ to a proper $(\Delta+1)$-edge coloring with fewer edges colored $\Delta+1$.

If $d_{G_{\Delta}}\left(a_{i-1}\right)>1$, we consider a maximal $g_{i-1}$-fan $F=\left(e_{1}, e_{2}, \ldots, e_{k}\right)$ at $a_{i-1}$ with $e_{1}=a_{i-1} a_{i-2}$ and $e_{j}=a_{i-1} u_{j}$, for $j=2, \ldots, k$. If there is some color $c_{k+1} \neq \Delta+1$ missing at $u_{k}$ under $g_{i-1}$, then by proceeding similarly as in Case A we may transform $g_{i-1}$ to a proper $(\Delta+1)$-edge coloring $\alpha$ using only interchanges, where $\alpha$ satisfies $|M(\alpha, \Delta+1)|<|M(f, \Delta+1)|$.

So suppose that $\Delta+1$ is the only color that does not appear at $u_{k}$ under $g_{i-1}$. Note that this implies that $u_{k} \in V\left(G_{\Delta}\right)$ Additionally, $u_{k}$ is distinct from $a_{0}, a_{1}, \ldots, a_{i-1}$, because $u_{k} \neq a_{i-2}$ and $G_{\Delta}$ is acyclic.

We will now make a series of interchanges to obtain $g_{i}$ from $g_{i-1}$. Suppose that $g_{i-1}\left(e_{j}\right)=c_{j}, j=1, \ldots, k$, where $c_{1}=\Delta+1$. We first interchange colors on the maximal $\left(\Delta+1, c_{2}\right)$-colored path with origin at $a_{i-2}$ and denote the obtained coloring by $f_{1}$. Since the first edge of this path is colored $\Delta+1$, $\left|M\left(f_{1}, \Delta+1\right)\right| \leq\left|M\left(g_{i-1}, \Delta+1\right)\right|$. Now, for each $j=2, \ldots, k-1$, from $f_{i-1}$ we 
construct the coloring $f_{i}$ by interchanging colors on the maximal $\left(\Delta+1, c_{i+1}\right)$-colored path with origin at $u_{i}$. All these paths begin by an edge colored $\Delta+1$, so we do not increase the number of edges colored $\Delta+1$ in $G$ by making these interchanges. Note that $f_{k-1}\left(a_{i-1} u_{k}\right)=\Delta+1$. We set $g_{i}=f_{k-1}$ and $a_{i}=u_{k}$, and go to Step $(i+1)$.

Since $G_{\Delta}$ is acyclic, the algorithm above will stop after a finite number of steps, when we reach a vertex of degree 1 in $G_{\Delta}$. Moreover, the algorithm produces a proper $(\Delta+1)$ edge coloring $g$ of $G$ such that $|M(g, \Delta+1)| \leq|M(f, \Delta+1)|$, and there is an edge $e \in$ $E\left(G_{\Delta}\right) \cap M(g, \Delta+1)$ which is incident to a vertex of degree $1 \mathrm{in} G_{\Delta}$. Hence, this case has been reduced to Case A, and thus we may, using only interchanges, from $g$ construct a proper $(\Delta+1)$-edge coloring with fewer edges colored $\Delta+1$.

Case B.2 $E\left(G_{\Delta}\right) \cap M(f, \Delta+1)=\emptyset$ but some edge colored $\Delta+1$ is incident to a vertex of degree $\Delta$.

Let $e_{1}=u_{1} v$ be an edge colored $\Delta+1$ and suppose that $d_{G}(v)=\Delta$. We consider a maximal fan $F=\left(e_{1}, e_{2}, \ldots, e_{k}\right)$, at $v$ with $e_{i}=v u_{i}$, for $i=1, \ldots, k$. If there is a color $c \neq \Delta+1$ missing at $u_{k}$, then we proceed as in Case $\mathrm{A}$ and construct a proper $(\Delta+1)$-edge coloring $\alpha$ from $f$ via interchanges, such that $|M(\alpha, \Delta+1)|<|M(f, \Delta+1)|$.

So suppose $\Delta+1$ is the only color missing at $u_{k}$ under $f$. This implies that $u_{k} \in V\left(G_{\Delta}\right)$. We may now proceed exactly as described in Step $i$ of the algorithm above: by making a series of interchanges on maximal bicolored paths (where $\Delta+1$ is one of the colors on every such path), we obtain a proper $(\Delta+1)$-edge coloring $g$ of $G$ such that $|M(g, \Delta+1)| \leq|M(f, \Delta+1)|$ and $g\left(v u_{k}\right)=\Delta+1$; that is, there is an edge colored $\Delta+1$ under $g$ both ends of which have degree $\Delta$. Then the edge $e=v u_{k}$ satisfies the conditions of Case A or Case B.1; as before, we can construct a proper $(\Delta+1)$-edge coloring with fewer edges colored $\Delta+1$ than under $g$.

Case B.3 $E\left(G_{\Delta}\right) \cap M(f, \Delta+1)=\emptyset$ and no edge colored $\Delta+1$ is incident to a vertex of degree $\Delta$.

Suppose that $e_{1}=u_{1} v$ is colored $\Delta+1$ under $f$, and consider a maximal fan $F=\left(e_{1}, e_{2}, \ldots, e_{k}\right)$ at $v$, where $e_{i}=v u_{i}$, for $i=1, \ldots, k$. If there is a color $c \neq \Delta+1$ missing at $u_{k}$, then we proceed as in Case $\mathrm{A}$ and construct a proper $(\Delta+1)$-edge coloring $\alpha$ from $f$ via interchanges, such that $|M(\alpha, \Delta+1)|<|M(f, \Delta+1)|$.

So assume that $\Delta+1$ is the only color missing at $u_{k}$. Then $u_{k} \in V\left(G_{\Delta}\right)$ and we may now proceed as in Step $i$ of the algorithm above for obtaining a proper $(\Delta+1)$-edge coloring $g$ of $G$ from $f$, such that $|M(g, \Delta+1)| \leq|M(f, \Delta+1)|$ and there is an edge $e$ colored $\Delta+1$ under $g$ such that one end of $e$ is $u_{k}$, and thus has degree $\Delta$. Hence, this case has been reduced to Case B.2. This completes the proof of Proposition 2.1.

Let us now prove Theorem 1.1.

Proof of Theorem 1.1. If for every graph $G$ with $\Delta(G) \leq k$ all proper $\left(\chi^{\prime}(G)+1\right)$-edge colorings of $G$ are Kempe equivalent, then, using Theorem A, it is not difficult to show that every Class 1 graph $H$ with $\Delta(H) \leq k$ has the Vizing property. 
Conversely, assume that all Class 1 graphs with maximum degree at most $k$ have the Vizing property. We will prove that for each graph $G$ with $\Delta(G) \leq k$ all proper $\left(\chi^{\prime}(G)+1\right)$ edge colorings of $G$ are Kempe equivalent. The proof is by induction on $k$.

The proposition is evident for $k=2$. Suppose that the proposition is true for all integers less than $k$, and let $G$ be an arbitrary graph with $\Delta(G)=k$ and $\chi^{\prime}(G)=\chi^{\prime}$. Consider a proper $\chi^{\prime}$-edge coloring $h$ of $G$. We assume that $M\left(h, \chi^{\prime}\right)$ is a maximal matching of $G$. Otherwise we can consider instead of $h$ another proper $\chi^{\prime}$-edge coloring which can be obtained from $h$ by a sequence of interchanges as follows: if there is an edge $x y$ such that the color $\chi^{\prime}$ is missing at $x$ and at $y$, then recolor the edge $x y$ with color $\chi^{\prime}$. We repeat this procedure until we obtain a proper $\chi^{\prime}$-edge coloring where the edges colored $\chi^{\prime}$ form a maximal matching.

In order to show that any two proper $\left(\chi^{\prime}+1\right)$-edge colorings of $G$ are Kempe equivalent it is sufficient to show that any proper $\left(\chi^{\prime}+1\right)$-edge coloring of $G$ can be transformed to $h$ by a sequence of interchanges.

Let $\varphi$ be a proper $\left(\chi^{\prime}+1\right)$-edge coloring of $G$. First we transform $\varphi$ by a sequence of interchanges to a proper $\chi^{\prime}$-edge coloring. In the case when $G$ is a Class 1 graph it is possible because $G$ has the Vizing property, and in the case when $G$ is a Class 2 graph it is possible by Theorem A. The obtained coloring we denote by $\varphi_{1}$.

Then the coloring $\varphi_{1}$ can be transformed to a proper $\left(\chi^{\prime}+1\right)$-edge coloring by sequentially recoloring the edges in the matching $M\left(h, \chi^{\prime}\right)$ with color $\chi^{\prime}+1$. Clearly, the obtained proper $\left(\chi^{\prime}+1\right)$-coloring $f$ satisfies the condition $M\left(h, \chi^{\prime}\right)=M\left(f, \chi^{\prime}+1\right)$. Furthermore, $f$ is obtained from $\varphi$ by a sequence of interchanges.

Consider the graph $G_{1}=G-M\left(h, \chi^{\prime}\right)$. The proper $\chi^{\prime}(G)$-edge coloring $h$ of $G$ induces a proper $\left(\chi^{\prime}(G)-1\right)$-edge coloring $h^{\prime}$ of $G_{1}$ and the proper $\left(\chi^{\prime}(G)+1\right)$-edge coloring $f$ of $G$ induces a proper $\chi^{\prime}(G)$-edge coloring $f^{\prime}$ of $G_{1}$. Clearly, $\chi^{\prime}\left(G_{1}\right)=\chi^{\prime}(G)-1$. Therefore, $h^{\prime}$ is a proper $\chi^{\prime}\left(G_{1}\right)$-edge coloring and $f^{\prime}$ is a proper $\left(\chi^{\prime}\left(G_{1}\right)+1\right)$-edge coloring of $G_{1}$.

If $\Delta\left(G_{1}\right)=k-1$ then, by the induction hypothesis, $f^{\prime}$ and $h^{\prime}$ are Kempe equivalent. Then the coloring $\varphi$ of $G$ can be transformed to the coloring $h$ by a sequence of interchanges.

Suppose now that $\Delta\left(G_{1}\right)=k$. This is possible only if $G$ is a Class 2 graph and $G_{1}$ is a Class 1 graph, that is, if $\chi^{\prime}(G)=k+1$ and $\chi^{\prime}\left(G_{1}\right)=k$. Since $M(h, k+1)$ is a maximal matching in $G$, every two vertices of degree $k$ in $G_{1}$ are nonadjacent. By Proposition 2.1 the coloring $f^{\prime}$ can be transformed by interchanges to a proper $(k+1)$-edge coloring $f^{\prime \prime}$ such that $M\left(f^{\prime \prime}, k+1\right)=\emptyset$. Let $g$ be the proper $(k+1)$-edge coloring obtained from $f^{\prime \prime}$ by sequentially recoloring the edges in the set $M\left(h^{\prime}, k\right)$ with color $k+1$. Clearly, $g$ is obtained from $f^{\prime \prime}$ by a sequence of interchanges and $M(g, k+1)=M\left(h^{\prime}, k\right)$. Then the graph $G_{2}=G_{1}-M\left(h^{\prime}, k\right)$ has the maximum degree $k-1$. Let $h_{1}$ be the proper $(k-1)$-edge coloring of $G_{2}$ induced by the matchings $M\left(h^{\prime}, 1\right), M\left(h^{\prime}, 2\right), \ldots, M\left(h^{\prime}, k-1\right)$ and $g_{1}$ be the proper $k$-edge coloring of $G_{2}$ induced by the matchings $M(g, 1), M(g, 2), \ldots, M(g, k)$. By the induction hypothesis, $g_{1}$ can be transformed to $h_{1}$ by a sequence of interchanges. Then the coloring $\varphi$ also can be transformed to $h$ by a sequence of interchanges.

We have thus proved that for every graph $G$ with $\Delta(G)=k$ all proper $\left(\chi^{\prime}(G)+1\right)$-edge colorings of $G$ are Kempe equivalent. The proposition follows by the principle of induction.

Next, we will prove Theorem 1.2, The proof is similar to the proof of Theorem 1.1.

Proof of Theorem 1.2. Assume that for every graph $H$ with $\Delta(H)=k-1$ all proper 
$\left(\chi^{\prime}(H)+1\right)$-edge colorings of $H$ are Kempe equivalent, and let $G$ be a Class 2 graph with $\Delta(G)=k$ and $\chi^{\prime}(G)=k+1$.

Consider a proper $(k+1)$-edge coloring $h$ of $G$. Again as in the proof of Theorem 1.1 we may assume that $M(h, k+1)$ is a maximal matching of $G$. Our goal is then to show that any proper $(k+2)$-edge coloring of $G$ can be transformed to $h$ by a sequence of interchanges.

Let $\varphi$ be a proper $(k+2)$-edge coloring of $G$. By Theorem A, we can transform $\varphi$ by a sequence of interchanges to a proper $(k+1)$-edge coloring. The obtained coloring we denote by $\varphi_{1}$.

Then the coloring $\varphi_{1}$ can be transformed to a proper $(k+2)$-edge coloring by sequentially recoloring the edges in the matching $M(h, k+1)$ with color $k+2$. Clearly, the obtained proper $(k+2)$-coloring $f$ satisfies the condition $M(h, k+1)=M(f, k+2)$. Furthermore, $f$ is obtained from $\varphi$ by a sequence of interchanges.

Consider the graph $G_{1}=G-M(h, k+1)$. The proper $(k+1)$-edge coloring $h$ of $G$ induces a proper $k$-edge coloring $h^{\prime}$ of $G_{1}$ and the proper $(k+2)$-edge coloring $f$ of $G$ induces a proper $(k+1)$-edge coloring $f^{\prime}$ of $G_{1}$. Clearly, $\chi^{\prime}\left(G_{1}\right)=k$. Therefore, $h^{\prime}$ is a proper $\chi^{\prime}\left(G_{1}\right)$-edge coloring and $f^{\prime}$ is a proper $\left(\chi^{\prime}\left(G_{1}\right)+1\right)$-edge coloring of $G_{1}$.

If $\Delta\left(G_{1}\right)=k-1$, then $G_{1}$ is Class 2 , and it follows from our assumption that $f^{\prime}$ can be transformed to $h^{\prime}$ by a sequence of interchanges. Then the coloring $\varphi$ of $G$ also can be transformed to the coloring $h$ by a sequence of interchanges.

In the case when $\Delta\left(G_{1}\right)=k$, the argument is the same as in the proof of Theorem 1.1 with only one difference: instead of the induction hypothesis we use the above assumption of the theorem. The details are omitted.

\section{Proof of Theorem 1.3}

In this section we will prove the main result of our paper. First we prove the result for 4-regular graphs (see Theorem 3.4 below) and then, using it, for any graph with maximum degree 4.

The following three lemmas are fundamental for our proofs of Theorems 1.3 and 3.4 .

Lemma 3.1. Let $G$ be a 4-regular graph, $f$ be a proper 5-edge coloring of $G$ and assume that $P$ is a $(1,2)$-colored path under $f$ in $G$.

(a) Suppose that $P$ has length 4 and the internal vertices along $P$ are $v_{1}, v_{2}, v_{3}$. Assume further that at least one of the following conditions does not hold:

(i) $\left|f\left(v_{i}\right) \cap f\left(v_{j}\right) \backslash\{1,2\}\right|=1$ for $i, j \in\{1,2,3\}$ satisfying $i \neq j$,

(ii) if $x_{1}$ and $x_{2}$ are the vertices distinct from $v_{1}$ and $v_{3}$ that are adjacent to $v_{2}$, then colors $3,4,5$ appear at $x_{1}$ and $x_{2}$ under $f$.

Then there is a proper 5-edge coloring $g$ of $G$ which can be obtained from $f$ by a sequence of interchanges on bicolored paths with colors from $\{3,4,5\}$, such that one of colors $3,4,5$ is missing either in the set $g\left(v_{1}\right) \cup g\left(v_{2}\right)$ or in $g\left(v_{2}\right) \cup g\left(v_{3}\right)$. 
(b) If $P$ has length 5 and $v_{1}, v_{2}, v_{3}, v_{4}$ are the internal vertices along $P$, then there is a proper 5 -edge coloring $g$ of $G$ which can be obtained from $f$ by a sequence of interchanges on bicolored paths with colors from $\{3,4,5\}$, such that one of colors $3,4,5$ is missing in the set $g\left(v_{i}\right) \cup g\left(v_{i+1}\right)$, for some $i \in\{1,2,3\}$.

Proof. We first prove (a). By the definition of the path $P,\{1,2\} \subseteq f\left(v_{i}\right)$, for $i=1,2,3$. We show that if the conclusion is false, then (i) and (ii) hold. So suppose that the conclusion of the lemma is false. Then $\{3,4,5\} \subseteq f\left(v_{1}\right) \cup f\left(v_{2}\right)$ and $\{3,4,5\} \subseteq f\left(v_{2}\right) \cup f\left(v_{3}\right)$, since otherwise the conclusion follows immediately by setting $g=f$. These two properties together with the conditions $\Delta(G)=4$ and $\{1,2\} \subseteq f\left(v_{i}\right)$, for $i=1,2,3$, imply that $\left|f\left(v_{1}\right) \cap f\left(v_{2}\right) \backslash\{1,2\}\right| \leq 1$ and $\left|f\left(v_{2}\right) \cap f\left(v_{3}\right) \backslash\{1,2\}\right| \leq 1$. Since $G$ is 4-regular, we thus have $\left|f\left(v_{1}\right) \cap f\left(v_{2}\right) \backslash\{1,2\}\right|=1$ and $\left|f\left(v_{2}\right) \cap f\left(v_{3}\right) \backslash\{1,2\}\right|=1$.

We now prove that $\left|f\left(v_{1}\right) \cap f\left(v_{3}\right) \backslash\{1,2\}\right|=1$. Suppose that this is not true, that is, $\left|f\left(v_{1}\right) \cap f\left(v_{3}\right) \backslash\{1,2\}\right|=2$. Without loss of generality we may assume that $f\left(v_{1}\right)=f\left(v_{3}\right)=$ $\{1,2,3,5\}$. Then $f\left(v_{2}\right)=\{1,2,4,5\}$ because $\{3,4,5\} \subseteq f\left(v_{2}\right) \cup f\left(v_{3}\right)$. Consider a maximal path $Q$ that is $(3,4)$-colored under $f$ and has origin at $v_{2}$. If $v_{1}$ is not an endpoint of this path, then color 4 neither appears at $v_{1}$ nor at $v_{2}$ after an interchange on $Q$; if $v_{1}$ is an endpoint of $Q$, then color 4 neither appears at $v_{2}$ nor at $v_{3}$ after an interchange on $Q$. In both cases the conclusion of the lemma holds; a contradiction. Hence, $\left|f\left(v_{1}\right) \cap f\left(v_{3}\right) \backslash\{1,2\}\right|=1$.

Suppose now that (i) holds but not (ii). Without loss of generality we can assume that

$$
f\left(v_{1}\right)=\{1,2,3,5\}, f\left(v_{2}\right)=\{1,2,4,5\}, f\left(v_{3}\right)=\{1,2,3,4\},
$$

and that $f\left(x_{1} v_{2}\right)=5$. If color 3 does not appear at $x_{1}$, then we may recolor the edge $v_{2} x_{1}$ with color 3 , obtaining a coloring where 5 does not appear at $v_{2}$ or $v_{3}$; a contradiction. Similarly, color 3 must appear at $x_{2}$. Hence, $\{3,5\} \subseteq f\left(x_{1}\right)$ and $\{3,4\} \subseteq f\left(x_{2}\right)$.

Let us now prove that color 4 appears at $x_{1}$. Consider a maximal $(3,4)$-colored path $Q$ with origin $v_{1}$. If $v_{2}$ is not an endpoint of this path, then by interchanging colors on $Q$ we get a coloring where 3 does not appear at $v_{1}$ or $v_{2}$. So suppose that $v_{2}$ is an endpoint of $Q$, interchange colors on $Q$ and denote the obtained coloring by $f_{1}$. If 4 does not appear at $x_{1}$ under $f_{1}$, then we may recolor $v_{2} x_{1}$ with color 4 to obtain a coloring where 5 does not appear at $v_{2}$ or $v_{3}$. Hence, $4 \in f\left(x_{1}\right)$. That color 5 must appear at $x_{2}$ under $f$ can be proved similarly by considering a maximal $(3,5)$-colored path with origin at $v_{3}$. Thus $\{3,4,5\} \subseteq f\left(x_{i}\right), i=1,2$.

We now prove statement (b) of Lemma 3.1. By part (a), we may assume that condition (i) holds for the vertices $v_{1}, v_{2}, v_{3}$, since otherwise the required result follows. In fact, we will as before assume (without loss of generality) that

$$
f\left(v_{1}\right)=\{1,2,3,5\}, f\left(v_{2}\right)=\{1,2,4,5\}, f\left(v_{3}\right)=\{1,2,3,4\} .
$$

If $f\left(v_{4}\right)=\{1,2,3,4\}$, then the result follows by setting $g=f$. If $f\left(v_{4}\right)=\{1,2,4,5\}$, then the result follows by applying part (a) to the subpath of $P$ with internal vertices $v_{2}, v_{3}, v_{4}$. So we may assume that $f\left(v_{4}\right)=\{1,2,3,5\}$. Consider a maximal $(3,4)$-colored path $Q$ with origin at $v_{2}$. Let $f_{1}$ be a proper 5 -edge coloring obtained from $f$ by interchanging colors on $Q$. If $v_{1}$ is not an endpoint of $Q$, then the result follows by setting $g=f_{1}$ because $4 \notin f_{1}\left(v_{1}\right) \cup f_{1}\left(v_{2}\right)$. Suppose now that $v_{1}$ is an endpoint of $Q$. Then $\left|f_{1}\left(v_{2}\right) \cap f_{1}\left(v_{4}\right) \backslash\{1,2\}\right|=2$. The result now follows by applying part (a) to the subpath of $P$ with internal vertices $v_{2}, v_{3}, v_{4}$. 
Lemma 3.2. Let $G$ be a 4-regular graph with $\chi^{\prime}(G)=4, f$ be a proper 5-edge coloring of $G$, $h$ a proper 4-edge coloring of $G$, and let $P=u_{1} v_{1} v_{2} v_{3} v_{4}$ be a $(1,2)$-colored path under $f$ in $G$ such that $f\left(u_{1} v_{1}\right)=2$ and $h\left(u_{1} v_{1}\right)=1$. Assume further that

$$
f\left(v_{1}\right)=\left\{1,2, c_{1}, c_{3}\right\}, f\left(v_{2}\right)=\left\{1,2, c_{2}, c_{3}\right\}, f\left(v_{3}\right)=\left\{1,2, c_{1}, c_{2}\right\},
$$

where $\left(c_{1}, c_{2}, c_{3}\right)$ is a permutation of the set $\{3,4,5\}$. Let $x_{1}$ and $x_{2}$ be the vertices distinct from $v_{1}$ and $v_{3}$ that are adjacent to $v_{2}$, where $f\left(v_{2} x_{1}\right)=c_{3}, f\left(v_{2} x_{2}\right)=c_{2}$. If $u_{1} \neq x_{2}$, then one of the following holds:

(I) $f\left(x_{1}\right)=\{2,3,4,5\}$ and $f\left(x_{2}\right)=\{1,3,4,5\}$,

(II) there is a proper 5-edge coloring $g$ of $G$ which can be obtained from $f$ by a sequence of interchanges, such that $M(h, 1) \cap M(g, 1)=M(h, 1) \cap M(f, 1)$, the color 1 is missing in $g\left(v_{1}\right)$ and, moreover, if $1 \notin f\left(u_{1}\right)$, then $1 \notin g\left(u_{1}\right)$,

(III) there is a proper 5 -edge coloring $g$ of $G$ which can be obtained from $f$ by a sequence of interchanges on bicolored paths with colors from $\{3,4,5\}$, such that one of colors $3,4,5$ is missing either in the set $g\left(v_{1}\right) \cup g\left(v_{2}\right)$ or in $g\left(v_{2}\right) \cup g\left(v_{3}\right)$.

Proof. Suppose that (III) does not hold. Then, by Lemma 3.1, colors 3, 4, 5 appear at both $x_{1}$ and $x_{2}$ under $f$.

First we show that $f\left(x_{2}\right)=\{1,3,4,5\}$ or (II) holds. Suppose that $1 \notin f\left(x_{2}\right)$. Then $v_{1} v_{2} x_{2}$ is a maximal $\left(1, c_{2}\right)$-colored path under $f$. Since, $u \neq x_{2}$ we may interchange colors on this path to obtain a proper 5-edge coloring $g$ of $G$ for which (II) holds. Hence we may assume that $1 \in f\left(x_{2}\right)$, that is, $f\left(x_{2}\right)=\{1,3,4,5\}$.

Now we show that $f\left(x_{1}\right)=\{2,3,4,5\}$ or (II) holds. Suppose that $2 \notin f\left(x_{1}\right)$. Then the sequence $\left(v_{2} v_{1}, v_{2} x_{2}, v_{2} v_{3}, v_{2} x_{1}\right)$ is an $f$-fan at $v_{2}$ and color 2 does not appear at $x_{1}$ and $x_{2}$. Note also that $c_{1}$ does not appear at $v_{2}$. Consider a maximal path $Q$ that is $\left(c_{1}, 2\right)$-colored under $f$ and has origin at $x_{1}$.

If $\left\{v_{3}, x_{2}\right\} \cap V(Q)=\emptyset$, then we interchange colors on $Q$ and obtain a proper coloring that we denote by $f_{1}$. The sequence $\left(v_{2} v_{1}, v_{2} x_{2}, v_{2} v_{3}, v_{2} x_{1}\right)$ is a saturated $f_{1}$-fan at $v_{2}$ and we may downshift to obtain a proper 5 -edge coloring $g$ under which color 1 does not appear at $v_{1}$. Moreover, if $1 \notin f\left(u_{1}\right)$, then $1 \notin g\left(u_{1}\right)$; that is, (II) holds.

Suppose now that $v_{3} \in V(Q)$. Then $x_{2} \notin V(Q)$. Consider a maximal path $Q^{\prime}$ that is $\left(c_{1}, 2\right)$-colored under $f$ and has origin $x_{2}$. Clearly, $Q$ and $Q^{\prime}$ are disjoint and by interchanging colors on $Q^{\prime}$ we get a coloring $f_{1}$ where $\left(v_{2} v_{1}, v_{2} x_{2}\right)$ is a saturated $f_{1}$-fan at $v_{2}$. By downshifting on this fan we obtain a coloring $g$ for which (II) holds.

Suppose now that $x_{2} \in V(Q)$. Then $v_{3} \notin V(Q)$ and by interchanging colors on $Q$ we get a coloring $f_{1}$ where $\left(v_{2} v_{1}, v_{2} x_{2}\right)$ is a saturated $f_{1}$-fan. Again, downshifting on this fan yields that (II) holds.

Let $G$ be a graph with $\chi^{\prime}(G)=\Delta(G)=4$ and $h$ a proper 4-edge coloring of $G$. For an arbitrary proper 5-edge coloring $\varphi$ of $G$ we say that an edge $e$ is $h$-correct under $\varphi$ (or just correct under $\varphi$ ) if $\varphi(e)=h(e)$. By the distance between two edges $e$ and $e^{\prime}$ on a path $P$, we mean the number of edges between $e$ and $e^{\prime}$ on $P$. 
Lemma 3.3. Let $G$ be a graph with $\chi^{\prime}(G)=\Delta(G)=4$, $h$ be a proper 4-edge coloring of $G$ and $f$ a proper 5 -edge coloring of $G$. Furthermore, let $P$ be a maximal path or cycle that is $(1,2)$-colored under $f$ and which contains an edge $x y$ such that $f(x y)=2$ and $h(x y)=1$. If there is no h-correct edge of color 1 under $f$ at distance at most 2 from $x y$ on $P$, then there is a proper 5-edge coloring $f^{\prime}$ of $G$ such that $f^{\prime}$ can be obtained from $f$ by a sequence of interchanges and $\left|M\left(f^{\prime}, 1\right) \cap M(h, 1)\right|>|M(f, 1) \cap M(h, 1)|$.

Proof. Suppose first that $P$ contains no $h$-correct edge colored 1 . Then we simply make an interchange on $P$ to obtain the required coloring $f^{\prime}$.

Suppose now that $P$ contains some $h$-correct edge colored 1 . This implies that the length of $P$ is at least 6 .

Case 1. $P$ is a path and the distance between $x y$ and the first or the last edge of $P$ is at most 3 .

Let $P=a_{l} a_{l-1} \ldots a_{1} x y b_{1} \ldots b_{k}$, where $l \leq 4$ or $k \leq 4$. Without loss of generality we assume that $l \leq 4$. The conditions imply that the subpath $a_{l} a_{l-1} \ldots x$ contains no $h$-correct edge colored 1 under $f$, so we must have $k \geq 5$. Consider the path $y b_{1} b_{2} b_{3} b_{4} b_{5}$. By Lemma 3.1 (b), there is a sequence of interchanges on bicolored paths with colors from $\{3,4,5\}$ yielding a proper 5-edge coloring $g$ of $G$ from $f$, such that for some color $c \in\{3,4,5\}$ and some $i \in\{1,2,3\}$, we have $c \notin g\left(b_{i}\right) \cup g\left(b_{i+1}\right)$. Note that $b_{i} b_{i+1}$ is not both $h$-correct and colored 1. Recolor the edge $b_{i} b_{i+1}$ with the color $c$ and denote the obtained coloring by $g_{1}$. Then the path $Q=a_{l} a_{l-1} \ldots a_{1} x y b_{1} \ldots b_{i}$ is a maximal $(1,2)$-colored path under $g_{1}$ and it contains no correct edge colored 1 , so the desired result follows by making an interchange on $Q$.

Case 2. $P$ is a path and the distance between $x y$ and each of the first and last edges of $P$ is at least 4 .

Let $P=a_{l} a_{l-1} \ldots a_{1} x y b_{1} \ldots b_{k}$, where $l, k \geq 5$. Consider the path $y b_{1} b_{2} b_{3} b_{4} b_{5}$. By Lemma 3.1 (b), there is a sequence of interchanges on bicolored paths with colors from $\{3,4,5\}$ yielding a proper 5-edge coloring $g$ of $G$ from $f$, such that for some color $c \in\{3,4,5\}$ and some $i \in\{1,2,3\}$, we have $c \notin g\left(b_{i}\right) \cup g\left(b_{i+1}\right)$. Recolor the edge $b_{i} b_{i+1}$ with the color $c$ and denote the obtained coloring by $g_{1}$. Then the maximal path $Q$ that is $(1,2)$-colored under $g_{1}$ and contains $x y$, satisfies the condition of Case 1. Therefore, the coloring $g_{1}$ can be transformed to a required coloring $f^{\prime}$ by a sequence of interchanges. Thus, the lemma is true in Case 2 .

Case 3. $P$ is a cycle:

Suppose that $P=a_{k} a_{k-1} \ldots a_{1} x y b_{1} \ldots b_{k} a_{k}$. If $P$ has length at most 8 , then it contains no $h$-correct edge colored 1 , which contradicts our assumption; so assume that $P$ has length at least 10. If $P$ has length 10 , then there is at most one $h$-correct edge colored 1 on $P$, namely $b_{4} a_{4}$. Suppose that $b_{4} a_{4}$ is $h$-correct. By applying Lemma 3.1 (b) to the path $y b_{1} b_{2} b_{3} b_{4} a_{4}$, and proceeding as above we may reduce this situation to Case 1.

If, on the other hand, $P$ has length at least 12 , then there is a path $y b_{1} b_{2} b_{3} b_{4} b_{5}$ lying on $P$, and we may proceed exactly as in Case 2 for reducing this case to Case 1.

Theorem 3.4. Let $G$ be a 4-regular graph with $\chi^{\prime}(G)=4$. Then every proper 5 -edge coloring of $G$ can be transformed to any proper 4-edge coloring of $G$ by a sequence of interchanges. 
Proof. Let $h$ be a proper 4-edge coloring and $f$ be a proper 5-edge coloring of the graph $G$. It suffices to prove that the coloring $f$ can, by a sequence of interchanges, be transformed to a proper 5-edge coloring $g$ such that $M(g, 1)=M(h, 1)$. The result then follows by applying Theorem B to the graph $G^{\prime}=G-M(g, 1)$ with maximum degree 3 and two proper edge colorings of $G^{\prime}$ induced by the colorings $g$ and $h$, respectively.

Suppose that there is some edge $e=u_{1} v_{1}$ of $G$ such that $h(e)=1 \neq f(e)$. Without loss of generality we assume that $f(e)=2$. We will prove that by a sequence of interchanges we can transform $f$ into a proper 5 -edge coloring $f^{\prime}$ which satisfies

$$
\left|M\left(f^{\prime}, 1\right) \cap M(h, 1)\right|>|M(f, 1) \cap M(h, 1)| .
$$

This suffices for proving the theorem.

The remaining part of the proof proceeds by rather extensive case analysis, and we now give a brief outline. To begin with, we distinguish between the cases that $e$ is adjacent to at most one edge colored 1 under $f$ (Case A below) and the case when $e$ is adjacent to two edges colored 1 under $f$ (Case B below). In Case A the proof consists of several different subcases and the required coloring $f^{\prime}$ is constructed in different ways depending on the structure of the component of $G_{f}(1,2)$ containing $e$.

Case B also breaks into several different subcases, and here the analysis is considerably more involved. Several reductions are used, but we still have to consider quite a number of different cases depending on the structure of the component of $G_{f}(1,2)$ containing $e$. The general technique employed is to reduce different subcases to previously considered ones. Let us now give the details of the case analysis.

Case A. $e$ is adjacent to at most one edge colored 1 under $f$ :

Without loss of generality we assume that color 1 appears at $v_{1}$ under $f$ (if $e$ is not adjacent to any edge colored 1 , then we just recolor $e$ with 1 and are done). Consider a maximal path $P=u_{1} v_{1} v_{2} \ldots v_{k}$ in $G$ that is $(1,2)$-colored and has origin at $u_{1}$. Note that since $h\left(u_{1} v_{1}\right)=1$, $v_{1} v_{2}$ is not correct under $f$. If $k \leq 3$, then, by Lemma 3.3, we can transform $f$ by interchanges to a required coloring $f^{\prime}$ satisfying (3.1). Suppose now that $k \geq 4$. If $v_{3} v_{4}$ is not correct, then again by Lemma 3.3, we can transform $f$ by interchanges to a required coloring $f^{\prime}$ satisfying (3.1). So in the following we will assume that $v_{3} v_{4}$ is correct.

Case A.1. One of the conditions (i), (ii) in Lemma 3.1 does not hold.

Then there is a sequence of interchanges on bicolored paths with colors from $\{3,4,5\}$ which transform $f$ to a proper 5 -edge coloring $g$ such that $c \notin g\left(v_{i}\right) \cup g\left(v_{i+1}\right)$ for some color $c \in$ $\{3,4,5\}$ and some $i \in\{1,2\}$. Now we recolor $v_{i} v_{i+1}$ with $c$ and denote by $g_{1}$ the proper edge coloring obtained from this operation. Then the maximal $(1,2)$-colored path (under $g_{1}$ ) with origin at $u_{1}$ has length at most two and, by Lemma 3.3, $g_{1}$ can by interchanges be transformed into a proper 5-edge coloring $f^{\prime}$ such that $\left|M\left(f^{\prime}, 1\right) \cap M(h, 1)\right|>\left|M\left(g_{1}, 1\right) \cap M(h, 1)\right|$. This and $M\left(g_{1}, 1\right) \cap M(h, 1)=M(f, 1) \cap M(h, 1)$ imply that $\left|M\left(f^{\prime}, 1\right) \cap M(h, 1)\right|>|M(f, 1) \cap M(h, 1)|$.

Case A.2. The conditions (i),(ii) in Lemma 3.1 hold.

This means that $\left|f\left(v_{i}\right) \cap f\left(v_{j}\right) \backslash\{1,2\}\right|=1$ for $i, j \in\{1,2,3\}$ and $i \neq j$; and the colors $3,4,5$ appear at $x_{1}$ and $x_{2}$, where $x_{1}, x_{2}$ denote the vertices distinct from $v_{1}$ and $v_{3}$ that are 
adjacent to $v_{2}$. Then

$$
f\left(v_{1}\right)=\left\{1,2, c_{1}, c_{3}\right\}, f\left(v_{2}\right)=\left\{1,2, c_{2}, c_{3}\right\}, f\left(v_{3}\right)=\left\{1,2, c_{1}, c_{2}\right\}
$$

for some permutation $\left(c_{1}, c_{2}, c_{3}\right)$ of the set $\{3,4,5\}$. Clearly, $\left\{f\left(v_{2} x_{1}\right), f\left(v_{2} x_{2}\right)\right\}=\left\{c_{2}, c_{3}\right\}$. Let $f\left(v_{2} x_{1}\right)=c_{3}$ and $f\left(v_{2} x_{2}\right)=c_{2}$. Without loss of generality we may assume that

$$
f\left(v_{1}\right)=\{1,2,3,5\}, f\left(v_{2}\right)=\{1,2,4,5\}, f\left(v_{3}\right)=\{1,2,3,4\} .
$$

Then $f\left(v_{2} x_{1}\right)=5$ and $f\left(v_{2} x_{2}\right)=4$.

Case A.2.1. The vertex $v_{4}$ is an endpoint of $P$ and $x_{2} \neq u_{1}$.

First suppose that either $f\left(x_{1}\right) \neq\{2,3,4,5\}$ or $f\left(x_{2}\right) \neq\{1,3,4,5\}$. Then, by Lemma 3.2, either condition (II) or condition (III) of Lemma 3.2 holds.

If condition (III) holds, then arguing similarly as in Case A.1 we can by interchanges transform $f$ into a required coloring $f^{\prime}$ satisfying (3.1).

If condition (II) holds, then we can by interchanges transform $f$ into a proper 5-edge coloring $g$ such that $M(g, 1) \cap M(h, 1)=M(f, 1) \cap M(h, 1)$. Moreover, since color 1 does not appear at $u_{1}$ under $f$, we will have $1 \notin g\left(v_{1}\right), 1 \notin g\left(u_{1}\right)$. We color the edge $u_{1} v_{1}$ with color 1 and obtain a required coloring $f^{\prime}$ satisfying (3.1).

Now suppose that $f\left(x_{1}\right)=\{2,3,4,5\}$ and $f\left(x_{2}\right)=\{1,3,4,5\}$.

We first consider the case when $u_{1} \neq x_{1}$. We have that $f\left(v_{2} x_{1}\right)=5$. Clearly, $x_{1} \neq v_{4}$ because $1 \in f\left(v_{4}\right)$ and $f\left(x_{1}\right)=\{2,3,4,5\}$. We make an interchange on $P$ and denote the obtained coloring by $f_{1}$. Then $u_{1} v_{1}$ is correct under $f_{1}$, but $v_{3} v_{4}$ is not. Moreover, $x_{1} v_{2} v_{3}$ is a maximal $(5,1)$-colored path under $f_{1}$, and by interchanging colors on this path we get a new coloring which we denote by $f_{2}$. Since $x_{1} \neq v_{4}$, we may now recolor $v_{3} v_{4}$ with color 1 to obtain a required coloring $f^{\prime}$.

Suppose now that $u_{1}=x_{1}$. We have that $f\left(x_{2}\right)=\{1,3,4,5\}$ and $d_{G}\left(v_{2}\right)=4$. Then $v_{2}$ is incident to an edge colored 1 under $h$. Furthermore,

$$
h\left(x_{1} v_{1}\right)=h\left(v_{3} v_{4}\right)=1
$$

and thus we must have $h\left(v_{2} x_{2}\right)=1$. Let $x_{3}$ be the vertex adjacent to $x_{2}$, such that $f\left(x_{2} x_{3}\right)=$ 1. Since $h\left(v_{2} x_{2}\right)=1, x_{2} x_{3}$ is not correct under $f$, which implies that $v_{4} \notin\left\{x_{2}, x_{3}\right\}$. We now interchange colors on $P$ and denote the obtained coloring by $f_{1}$. Then $x_{1} v_{1}$ is correct under $f_{1}$, but $v_{3} v_{4}$ is not. Note that $v_{1} v_{2} x_{2}$ is a maximal $(4,2)$-colored path under $f_{1}$. We make an interchange on this path and denote the obtained coloring by $f_{2}$. Let $P^{\prime}=v_{4} v_{3} v_{2} x_{2} x_{3} \ldots$ be the maximal $(1,2)$-colored path under $f_{2}$ with origin at $v_{4}$. Since, $x_{2} x_{3}$ is not correct under $f$, it is not correct under $f_{2}$ too, and the desired result now follows by applying Lemma 3.3 to the coloring $f_{2}$, the path $P^{\prime}$ and the edge $v_{4} v_{3}$ instead of $f, P$ and $x y$.

Case A.2.2. The vertex $v_{4}$ is an endpoint of $P$ and $x_{2}=u_{1}$.

Recall that colors $3,4,5$ appear at $x_{1}$ under $f$. If 1 appears at $x_{1}$ under $f$, then the edge colored 1 incident to $x_{1}$ cannot be correct under $f$, because we must have $h\left(v_{2} x_{1}\right)=1$. This implies that $v_{4} \neq x_{1}$. Furthermore, $x_{1} v_{2} v_{3}$ is a maximal $(5,2)$-colored path under $f$ and by making an interchange on this path, we obtain a coloring that we denote by $f_{1}$. Now, note that $P^{\prime}=x_{2} v_{1} v_{2} x_{1} \ldots$ is a maximal $(1,2)$-colored path under $f_{1}$, and since the edge incident 
to $x_{1}$ colored 1 is not correct, it now follows from Lemma 3.3 that there is a proper 5-edge coloring $f^{\prime}$ of $G$ that can be obtained from $f_{1}$ via a sequence of interchanges, and such that $\left|M\left(f^{\prime}, 1\right) \cap M(h, 1)\right|>|M(f, 1) \cap M(h, 1)|$. If instead the color 2 appears at $x_{1}$ under $f$, then the proof proceeds exactly as in Case A.2.1 when $u_{1} \neq x_{1}$.

Case A.2.3. The vertex $v_{4}$ is an internal vertex of $P$.

It follows from Lemma 3.1 (b) that there is a sequence of interchanges on bicolored paths with colors from $\{3,4,5\}$ which transforms $f$ to a proper 5-edge coloring $g$ such that $c \notin$ $g\left(v_{i}\right) \cup g\left(v_{i+1}\right)$ for some color $c \in\{3,4,5\}$ and some $i \in\{1,2,3\}$.

If $i \leq 2$, then arguing similarly as in Case A.1, we can obtain a required coloring $f^{\prime}$ satisfying (3.1). Now we suppose that such a color $c$ exists only for $i=3$. This means that $c \notin g\left(v_{3}\right) \cup g\left(v_{4}\right),\{3,4,5\} \subseteq g\left(v_{1}\right) \cup g\left(v_{2}\right)$ and $\{3,4,5\} \subseteq g\left(v_{1}\right) \cup g\left(v_{2}\right)$.

If the coloring $g$ does not satisfy one of the conditions (i) and (ii) in Lemma 3.1 (with $g$ instead of $f$ ), then in a similar way as in Case A.1 we can obtain a required coloring $f^{\prime}$.

Suppose now that the conditions (i), (ii) of Lemma 3.1 for the coloring $g$ (instead of $f$ ) hold. Then

$$
g\left(v_{1}\right)=\left\{1,2, b_{1}, b_{3}\right\}, g\left(v_{2}\right)=\left\{1,2, b_{2}, b_{3}\right\}, g\left(v_{3}\right)=\left\{1,2, b_{1}, b_{2}\right\}
$$

for some permutation $\left(b_{1}, b_{2}, b_{3}\right)$ of the set $\{3,4,5\}$. Clearly, $\left\{f\left(v_{2} x_{1}\right), f\left(v_{2} x_{2}\right)\right\}=\left\{b_{2}, b_{3}\right\}$.

Without loss of generality we can assume that

$$
g\left(v_{1}\right)=\{1,2,3,5\}, g\left(v_{2}\right)=\{1,2,4,5\}, g\left(v_{3}\right)=\{1,2,3,4\},
$$

and that $g\left(x_{1} v_{2}\right)=5, g\left(x_{2} v_{2}\right)=4$ (possibly renaming the vertices $x_{1}$ and $x_{2}$ if this does not hold). Since there is a color $c \notin g\left(v_{3}\right) \cup g\left(v_{4}\right)$ and $g\left(v_{3}\right)=\{1,2,3,4\}$, we have that $c=5$ and $g\left(v_{4}\right)=\{1,2,3,4\}$. If $u_{1} \neq x_{2}$, then similarly as in Case A.2.1, it follows from Lemma 3.2 that either $g\left(x_{1}\right)=\{2,3,4,5\}$ and $g\left(x_{2}\right)=\{1,3,4,5\}$, or we can obtain a required coloring $f^{\prime}$ satisfying (3.1). Thus if $u_{1} \neq x_{2}$, then it suffices to consider the case when $g\left(x_{1}\right)=\{2,3,4,5\}$ and $g\left(x_{2}\right)=\{1,3,4,5\}$.

We first consider the case when $u_{1} \notin\left\{x_{1}, x_{2}\right\}$. From $g$ we costruct the coloring $g_{1}$ by recoloring $v_{3} v_{4}$ with color 5 . Then $g_{1}\left(x_{1}\right)=\{2,3,4,5\}$ and $g_{1}\left(x_{2}\right)=\{1,3,4,5\}$. Now we make an interchange on the path $u_{1} v_{1} v_{2} v_{3}$ and denote the constructed coloring by $g_{2}$. Note that $v_{4} v_{3} v_{2} x_{1}$ is a maximal $(1,5)$-colored path under $g_{2}$. By making an interchange on this path we obtain a required coloring $f^{\prime}$ satisfying (3.1).

Now assume that $u_{1}=x_{1}$. From $g$ we construct the coloring $g_{1}$ by recoloring $v_{3} v_{4}$ with color 5. Then $g_{1}\left(x_{1}\right)=\{2,3,4,5\}$ and $g_{1}\left(x_{2}\right)=\{1,3,4,5\}$. By making an interchange on the path $x_{1} v_{1} v_{2} v_{3}$ we obtain a coloring $g_{2}$. Note that the path $v_{4} v_{3} v_{2} x_{1} v_{1}$ is $(5,1)$-colored under $g_{2}$ and that the edge $v_{3} v_{4}$ is adjacent to only one edge colored 1 . Moreover, $v_{2}$ and $v_{4}$ are not adjacent, because $g\left(x_{1}\right) \neq g\left(v_{4}\right)$ and $g\left(x_{2}\right) \neq g\left(v_{4}\right)$. Consider the path $P^{\prime}=u_{1}^{\prime} v_{1}^{\prime} v_{2}^{\prime} v_{3}^{\prime} v_{4}^{\prime}$, where $u_{1}^{\prime}=v_{4}, v_{1}^{\prime}=v_{3}, v_{2}^{\prime}=v_{2}, v_{3}^{\prime}=u_{1}$ and $v_{4}^{\prime}=v_{1}$. If we exchange the colors 5 and 2 in the coloring $g_{2}$ and consider the obtained coloring instead of $g$, the path $P^{\prime}=u_{1}^{\prime} v_{1}^{\prime} v_{2}^{\prime} v_{3}^{\prime} v_{4}^{\prime}$ instead of $P$ and the vertices $x_{1}^{\prime}=v_{1}, x_{2}^{\prime}=x_{2}$ instead of $x_{1}$ and $x_{2}$, then we obtain a situation which is similar to the situation considered in the previous paragraph, because here $u_{1}^{\prime} \notin\left\{x_{1}^{\prime}, x_{2}^{\prime}\right\}$.

Now assume that $u_{1}=x_{2}$. Recall that colors $3,4,5$ appear at both $x_{1}$ and $x_{2}$ under $f$. As before, we construct the coloring $g_{1}$ from $g$ by recoloring $v_{3} v_{4}$ with color 5 . Thereafter, we make a interchange on the path $x_{2} v_{1} v_{2} v_{3}$ and denote the obtained coloring by $g_{2}$. 
If $x_{1}$ is incident to an edge colored 2 under $g_{2}$, then $v_{4} v_{3} v_{2} x_{1}$ is a maximal $(1,5)$-colored path under $g_{2}$. By making an interchange on this path we obtain a required coloring $f^{\prime}$ satisfying (3.1).

Suppose now that $x_{1}$ is incident to an edge colored 1 under $g_{2}$. Consider the path $v_{4} v_{3} v_{2} x_{1}$ that is $(5,1)$-colored path under $g_{2}$ with origin at $v_{4}$. Clearly, the edge colored 1 incident to $x_{1}$ cannot be correct under $g_{2}$ (because we must have $h\left(x_{1} v_{2}\right)=1$ ). Put $x^{\prime}=v_{4}, y^{\prime}=v_{3}$ and let $P^{\prime}=v_{4} v_{3} v_{2} x_{1} \ldots$ be the maximal $(5,1)$-colored path with origin at $x^{\prime}=v_{4}$. Now we obtain the required result by applying Lemma 3.3 to the coloring $g_{2}$, the path $P^{\prime}$ and the edge $x^{\prime} y^{\prime}$ instead of $f, P$ and $x y$.

Case B. $e$ is adjacent to two edges colored 1 under $f$.

We have that $f(e)=2$. Let $u_{1} u_{2}$ and $v_{1} v_{2}$ be the edges adjacent to $e$ and colored 1 under $f$. Since $h(e)=1$, none of the edges $u_{1} u_{2}$ and $v_{1} v_{2}$ are $h$-correct. Let $P$ be the component in $G_{f}(1,2)$ that contains $e$. It can be a path or cycle.

If there is no correct edge colored 1 under $f$ at distance 2 from $e$ along $P$, then the theorem is true by Lemma 3.3 .

Suppose now that there is a correct edge at distance 2 from $e$ on $P$. This implies that if $P$ is a cycle then it has the length at least 6 .

Case B.1. $P$ is a path where the distance between $e$ and the first or the last edge of $P$ is at most 1 .

Let $P=u_{l} u_{l-1} \ldots u_{1} v_{1} v_{2} \ldots v_{k}$, where $2 \leq l \leq 3$ or $2 \leq k \leq 3$. Without loss of generality we may assume that $2 \leq l \leq 3$. Since there is a correct edge at distance 2 from $e$ on $P$, we will have that $k \geq 4$ and the edge $v_{3} v_{4}$ is correct. If one of the conditions (i) and (ii) of Lemma 3.1 does not hold, then arguing similarly as in Case A.1 we can by a sequence of interchanges transform $f$ to a proper coloring $f^{\prime}$ satisfying (3.1).

Assume now that the conditions (i), (ii) of Lemma 3.1 hold. Then we may assume without loss of generality that

$$
f\left(v_{1}\right)=\{1,2,3,5\}, f\left(v_{2}\right)=\{1,2,4,5\}, f\left(v_{3}\right)=\{1,2,3,4\}, f\left(v_{2} x_{1}\right)=5, f\left(v_{2} x_{2}\right)=4,
$$

and the colors $3,4,5$ appear at $x_{1}$ and $x_{2}$, where $x_{1}, x_{2}$ are the vertices distinct from $v_{1}$ and $v_{3}$ that are adjacent to $v_{2}$. Since $l \geq 2$, we have that $\{1,2,\} \subseteq f\left(u_{1}\right)$. Therefore $u_{1} \notin\left\{x_{1}, x_{2}\right\}$.

Suppose that $f\left(x_{1}\right) \neq\{2,3,4,5\}$. Then one of the conditions (II) and (III) of Lemma 3.2 holds. If the condition (II) holds, then we obtain a proper 5-edge coloring $g$ such that the color 1 is missing at $v_{1}$. This situation for $g$ is similar to the situation considered in Case A for $f$. Therefore we can transform $g$ by a sequence of interchanges to a required coloring $f^{\prime}$ satisfying (3.1).

If the condition (III) holds, then there is a sequence of interchanges on bicolored paths with colors from $\{3,4,5\}$ which transform $f$ to a proper 5-edge coloring $g$ such that $c \notin$ $g\left(v_{i}\right) \cup g\left(v_{i+1}\right)$ for some color $c \in\{3,4,5\}$ and some $i \in\{1,2\}$. Now we recolor $v_{i} v_{i+1}$ with $c$ and denote by $g_{1}$ the proper edge coloring obtained from this operation. Then the path $P^{\prime}=u_{l} u_{l-1} \ldots u_{1} v_{1} v_{2} \ldots v_{i}$ is the maximal $(1,2)$-colored (under $g_{1}$ ) path with origin at $u_{l}$, such that $g_{1}\left(u_{1} v_{1}\right)=2, h\left(u_{1} v_{1}\right)=1$ and there is no $h$-correct edge of color 1 on $P^{\prime}$. This situation for $g_{1}$ is similar to the situation considered in Lemma 3.3 for the coloring $f$, the 
path $P$ and the edge $x y$. Therefore we can transform $g_{1}$ by a sequence of interchanges to a required coloring $f^{\prime}$ satisfying (3.1).

We now consider the case when $f\left(x_{1}\right)=\{2,3,4,5\}$. If $v_{4}$ is an endpoint of $P$ then we proceed as follows: make an interchange on $P$ and denote the obtained coloring by $f_{1}$. Note that $\left|M\left(f_{1}, 1\right) \cap M(h, 1)\right|=|M(f, 1) \cap M(h, 1)|$, since $u_{1} v_{1}$ is correct under $f_{1}$, but $v_{3} v_{4}$ is not. However, $v_{3} v_{4}$ is adjacent to only one edge colored 1 under $f_{1}$. Thus we have a situation that is similar to the situation considered in Case $\mathrm{A}$ with the coloring $f_{1}$ and the edge $v_{3} v_{4}$ instead of $f$ and $e$. Hence we can by interchanges transform $f_{1}$, and therefore $f$ too, to a required coloring $f^{\prime}$ satisfying (3.1).

If $v_{4}$ is not an endpoint of $P$, then by Lemma 3.1(b) there is a sequence of interchanges on bicolored paths with colors from $\{3,4,5\}$ yielding a coloring $g$, such that $c \notin g\left(v_{j}\right) \cup g\left(v_{j+1}\right)$, for some $j \in\{1,2,3\}$ and some color $c \in\{3,4,5\}$. Recolor $v_{j} v_{j+1}$ with $c$ and denote the obtained coloring by $g_{1}$. If $j \leq 2$, the desired result follows by applying Lemma 3.3 to the coloring $g_{1}$ instead of $f$. Suppose now that $j=3$. We make an interchange on the maximal $(1,2)$-colored path containing $u_{1} v_{1}$ and denote the obtained coloring by $g_{2}$. Then $u_{1} v_{1}$ is correct under $g_{2}$ but $v_{3} v_{4}$ is not. Furthermore $\left|M\left(g_{2}, 1\right) \cap M(h, 1)\right| \geq|M(f, 1) \cap M(h, 1)|$ and $v_{3} v_{4}$ is adjacent to only one edge colored 1 under $g_{2}$. Thus we have a situation that is similar to the situation considered in Case A with the coloring $g_{2}$ and the edge $v_{3} v_{4}$ instead of $f$ and $e$. Therefore we can by using interchanges construct a required coloring $f^{\prime}$ satisfying (3.1).

Case B.2. $P$ is a path with $P=u_{l} u_{l-1} \ldots u_{2} u_{1} v_{1} v_{2} \ldots v_{k}$ where $l \geq 4$ and $k \geq 4$, or $P$ is a cycle of length at least 6 with $P=u_{l} u_{l-1} \ldots u_{1} v_{1} v_{2} \ldots v_{l}$, where $l \geq 3$.

Case B.2.1. At least one of the conditions (i), (ii) of Lemma 3.1 does not hold.

Then, by Lemma 3.1 (a), there is a sequence of interchanges on bicolored paths with colors from $\{3,4,5\}$ which transforms $f$ to a proper 5-edge coloring $g$, such that $c \notin g\left(v_{j}\right) \cup g\left(v_{j+1}\right)$ for some color $c \in\{3,4,5\}$ and some $j \in\{1,2\}$. We recolor $v_{j} v_{j+1}$ with color $c$. If $j=1$, we obtain a situation similar to the situation considered in Case A. If $j=2$, we obtain a situation similar to the situation considered in Case B.1. Hence in both cases we can transform $f$ to a proper coloring $f^{\prime}$ satisfying (3.1) by a sequence of interchanges.

Case B.2.2. At least one of the following conditions does not hold:

$$
\left|f\left(u_{i}\right) \cap f\left(u_{j}\right) \backslash\{1,2\}\right|=1 \text { for } i, j \in\{1,2,3\} \text { satisfying } i \neq j
$$

if $y_{1}$ and $y_{2}$ are the vertices distinct from $u_{1}$ and $u_{3}$ that are adjacent to $u_{2}$, then colors $3,4,5$ appear at $y_{1}$ and $y_{2}$.

Consider the (1,2)-colored path $v_{1} u_{1} u_{2} u_{3} u_{4}$. Arguing similarly as in the Case B.2.1, we can construct a required coloring $f^{\prime}$ satisfying (3.1) by a sequence of interchanges.

Case B.2.3. The conditions (i), (ii) of Lemma 3.1 hold for the vertices $v_{1}, v_{2}, v_{3}$ and the conditions (3.2), (3.3) hold for the vertices $u_{1}, u_{2}, u_{3}$. 
Suppose that it is impossible to transform the coloring $f$ to a required coloring $f^{\prime}$ satisfying (3.1) by using only interchanges.

We will show that then $f$ can be transformed via interchanges on bicolored paths with colors from $\{3,4,5\}$ to a proper 5 -edge coloring $f_{1}$ such that

$$
f_{1}\left(v_{1}\right)=f_{1}\left(u_{1}\right), \quad f_{1}\left(v_{2}\right)=f_{1}\left(u_{2}\right), \quad f_{1}\left(v_{3}\right)=f_{1}\left(u_{3}\right) .
$$

Without loss of generality we may assume that

$$
f\left(v_{1}\right)=\{1,2,3,5\}, f\left(v_{2}\right)=\{1,2,4,5\}, f\left(v_{3}\right)=\{1,2,3,4\} .
$$

Suppose first that $f\left(u_{1}\right) \neq f\left(v_{1}\right)$, e.g. that $f\left(u_{1}\right)=\{1,2,4,5\}$. (The case when $f\left(u_{1}\right)=$ $\{1,2,3,4\}$ is similar.) Then, by condition (3.2), $f\left(u_{1}\right) \neq f\left(u_{2}\right)$. This means that either $f\left(u_{2}\right)=\{1,2,3,5\}$ or $f\left(u_{2}\right)=\{1,2,3,4\}$.

If $f\left(u_{2}\right)=\{1,2,3,5\}$, then, by condition (3.2), $f\left(u_{3}\right)=\{1,2,3,4\}$. Consider a maximal $(3,4)$-colored path $Q$ with origin at $u_{1}$. We show that $u_{2}$ is an endpoint of $Q$. Assuming that $u_{2}$ is not an endpoint of $Q$ and interchanging the colors on $Q$ we obtain a coloring $f_{2}$ such that $f_{2}\left(u_{1}\right)=f_{2}\left(u_{2}\right)=\{1,2,3,5\}$. Then we may recolor $u_{1} u_{2}$ with color 4 and obtain a situation similar to the situation considered in Case A, which is impossible because in that case we can transform $f$ by interchanges to a required coloring $f^{\prime}$ satisfying (3.1). Thus, $u_{2}$ is an endpoint of $Q$. Then by interchanging colors on $Q$ we obtain the desired coloring $f_{1}$ satisfying (3.4).

Now suppose that $f\left(u_{2}\right)=\{1,2,3,4\}$. Then, by condition (3.2), $f\left(u_{3}\right)=\{1,2,3,5\}$. Let $Q$ be a maximal $(4,3)$-colored path with origin at $u_{1}$. Arguing similarly as in the preceding paragraph we obtain that $u_{3}$ is an endpoint of $Q$. By interchanging colors on $Q$ we get a coloring $f^{*}$. Next, we consider a maximal path $Q^{\prime}$ that is $(3,5)$-colored under $f^{*}$ and has origin at $u_{2}$. Arguing similarly as in the preceding paragraph we obtain that $u_{3}$ is an endpoint of $Q^{\prime}$. Now by interchanging colors on $Q^{\prime}$ we get the desired coloring $f_{1}$ satisfying (3.4).

Suppose now that $f\left(u_{1}\right)=f\left(v_{1}\right)=\{1,2,3,5\}$.

If $f\left(u_{2}\right)=f\left(v_{2}\right)=\{1,2,4,5\}$ then, by (3.2), $f\left(u_{3}\right)=\{1,2,3,4\}=f\left(v_{3}\right)$, that is, the coloring $f$ itself can be considered as the desired coloring $f_{1}$ satisfying (3.4).

If $f\left(u_{2}\right) \neq f\left(v_{2}\right)$ then the conditions (3.2) and (3.3) imply that $f\left(u_{2}\right)=\{1,2,3,4\}$ and $f\left(u_{3}\right)=\{1,2,4,5\}$. Let $Q_{1}$ be a maximal $(3,5)$-colored path with origin at $u_{2}$. Then $u_{3}$ is an endpoint of $Q_{1}$. (Otherwise by interchanging the colors on $Q_{1}$ we may obtain a coloring $f_{2}$ such that $f_{2}\left(u_{3}\right)=f_{2}\left(u_{2}\right)=\{1,2,4,5\}$. Then we may recolor $u_{2} u_{3}$ with color 3 and obtain a situation similar to the situation considered in Case B.1, which is impossible because in that case we can transform $f$ by interchanges to a required coloring $f^{\prime}$ satisfying (3.1).) Now by interchanging colors on $Q_{1}$ we obtain the desired coloring $f_{1}$ satisfying (3.4).

Thus we may, without loss of generality, further assume that $f$ can by interchanges on bicolored paths with colors from $\{3,4,5\}$ be transformed to a coloring $f_{1}$ such that

$$
f_{1}\left(v_{1}\right)=f_{1}\left(u_{1}\right)=\{1,2,3,5\}, f_{1}\left(v_{2}\right)=f_{1}\left(u_{2}\right)=\{1,2,4,5\}, f_{1}\left(v_{3}\right)=f_{1}\left(u_{3}\right)=\{1,2,3,4\} .
$$

Furthermore we can claim that it is impossible to transform $f_{1}$ to a proper 5-edge coloring $f^{\prime}$ satisfying (3.1) by using only interchanges, because $f_{1}$ is obtained from $f$ by a sequence 
of interchanges and, by our assumption, it is impossible to transform $f$ to a proper 5-edge coloring $f^{\prime}$ satisfying (3.1) by using only interchanges.

Denote by $x_{1}, x_{2}$ the vertices distinct from $v_{1}$ and $v_{3}$ that are adjacent to $v_{2}$, where $f\left(v_{2} x_{1}\right)=5$. We now prove that $f_{1}\left(x_{1}\right)=\{2,3,4,5\}$ and $f_{1}\left(x_{2}\right)=\{1,3,4,5\}$. Suppose that one of these two conditions does not hold. Then, by Lemma 3.2, one of conditions (II) and (III) of Lemma 3.2 holds. If the condition (III) holds, we may transform $f_{1}$ by interchanges on paths with colors from $\{3,4,5\}$ to a proper coloring $g$, such that $c \notin g\left(v_{i}\right) \cup g\left(v_{i+1}\right)$ for some color $c \in\{3,4,5\}$ and some $i \in\{1,2\}$. Then by recoloring $v_{i} v_{i+1}$ with $c$ we obtain a situation that is similar to the situation considered in Case A or Case B.1. If the condition (II) holds, then, by Lemma 3.2, we may transform $f_{1}$ by interchanges to another proper coloring and again obtain a situation which is similar to the situation considered in Case A.

Thus if one of conditions $f_{1}\left(x_{1}\right)=\{2,3,4,5\}$ and $f_{1}\left(x_{2}\right)=\{1,3,4,5\}$ does not hold, then we obtain situations which are similar to situations considered in Case A or Case B.1, which is impossible because in those cases we can transform $f_{1}$ by interchanges to a required coloring $f^{\prime}$ satisfying (3.1). Therefore $f_{1}\left(x_{1}\right)=\{2,3,4,5\}$ and $f_{1}\left(x_{2}\right)=\{1,3,4,5\}$.

Denote by $y_{1}$ and $y_{2}$ the vertices adjacent to $u_{2}$ that are distinct from $u_{1}$ and $u_{3}$, and where $f_{1}\left(u_{2} y_{1}\right)=5$. Clearly, $x_{1} \neq y_{1}$. Arguing similarly as in the preceding paragraph we may deduce that $f_{1}\left(y_{1}\right)=\{2,3,4,5\}$ and $f_{1}\left(y_{2}\right)=\{1,3,4,5\}$.

Hence, without loss of generality we will in the following assume that

$$
f_{1}\left(x_{1}\right)=f_{1}\left(y_{1}\right)=\{2,3,4,5\} \text { and } f_{1}\left(x_{2}\right)=f_{1}\left(y_{2}\right)=\{1,3,4,5\} .
$$

In particular, this implies that $x_{1}$ and $y_{1}$ are not internal vertices of $P$.

We will now show that $P$ is not a cycle of length 6 or a path of length 7 .

Suppose that $P$ is a cycle of length 6 , that is, $P=u_{3} u_{2} u_{1} v_{1} v_{2} v_{3} u_{3}$. Then the edge $v_{3} u_{3}$ is $h$-correct, because otherwise by interchanging colors on $P$ we can obtain a proper coloring $f^{\prime}$ satisfying (3.1), which is impossible. Using property (3.5) of $f_{1}$, we can obtain a proper coloring $f^{\prime}$ satisfying (3.1) by first recoloring $v_{3} u_{3}$ with color 5 , then interchanging colors on the path $v_{3} v_{2} v_{1} u_{1} u_{2} u_{3}$, and thereafter making an interchange on the maximal $(1,5)$-colored path $y_{1} u_{2} u_{3} v_{3} v_{2} x_{1}$. Since this is impossible, $P$ cannot be a cycle of length 6 .

Suppose now that $P$ is a path of length 7 . Then $P=u_{4} u_{3} u_{2} u_{1} v_{1} v_{2} v_{3} v_{4}$. Note that $\left\{v_{4}, u_{4}\right\} \cap\left\{x_{1}, y_{1}\right\}=\emptyset$, because $2 \notin f_{1}\left(v_{4}\right), 2 \notin f_{1}\left(u_{4}\right)$ and $2 \in f_{1}\left(x_{1}\right), 2 \in f_{1}\left(y_{1}\right)$. Make an interchange on $P$ and denote the obtained coloring by $f_{2}$. Then $u_{3} u_{2} y_{1}$ and $v_{3} v_{2} x_{1}$ are two disjoint maximal $(1,5)$-colored paths under $f_{2}$ in $G$. By interchanging colors on these paths and then recoloring $u_{4} u_{3}$ and $v_{4} v_{3}$ with color 1 we obtain a proper coloring $f^{\prime}$ satisfying (3.1). Since this is impossible, $P$ cannot be a path of length 7 .

Thus $P$ has the length at least 8 . We will show now that the edges $v_{3} v_{4}$ and $u_{3} u_{4}$ are $h$-correct under $f_{1}$.

Since $P$ has length at least 8 , at most one of $u_{4}$ and $v_{4}$ are endpoints of $P$. Suppose first that $u_{4}$ is an endpoint of $P$. If $u_{3} u_{4}$ is not correct, then we may proceed exactly as in Case B.1 to obtain a coloring $f^{\prime}$ satisfying (3.1), which contradicts our assumption that this is impossible. Hence, we may assume that $u_{3} u_{4}$ is correct.

If $v_{3} v_{4}$ is not correct, then by applying Lemma 3.1 (b) to the path $u_{1} v_{1} v_{2} v_{3} v_{4} v_{5}$ we get that there is a sequence of interchanges on bicolored paths with colors from $\{3,4,5\}$ yielding 
a coloring $g$, such that $c \notin g\left(v_{j}\right) \cup g\left(v_{j+1}\right)$ for some $j \in\{1,2,3\}$ and some color $c \in\{3,4,5\}$. By recoloring $v_{j} v_{j+1}$ with $c$, we obtain a situation which is similar to one of the situation considered in Case B.1 and Case A. This is impossible because in those cases we can obtain, by interchanges, a required coloring $f^{\prime}$ satisfying (3.1).

Suppose now that none of $u_{4}$ and $v_{4}$ is an endpoint of $P$. If $u_{3} u_{4}$ (or $v_{3} v_{4}$ ) is not correct, then by applying Lemma 3.1 (b) to the path $v_{1} u_{1} u_{2} u_{3} u_{4} u_{5}$ (or $u_{1} v_{1} v_{2} v_{3} v_{4} v_{5}$, respectively) and proceeding as in the preceding paragraph we obtain a situation which is similar to one of the situations considered in Case B.1 and Case A. This is impossible because in those cases we can obtain, by interchanges, a required coloring $f^{\prime}$ satisfying (3.1).

Thus we have that

the edges $u_{3} u_{4}$ and $v_{3} v_{4}$ are correct under $f_{1}$.

We now prove the following claim that holds for the coloring $f_{1}$ in Case B.2.3 when $P$ has the length at least 8 .

Claim 1. (a) If $Q$ is a maximal $(3,5)$-colored path under $f_{1}$ with origin at $u_{3}$ (at $\left.v_{3}\right)$, then $u_{2}$ (respectively, $v_{2}$ ) is an endpoint of $Q$.

(b) If $Q^{\prime}$ is a maximal $(4,5)$-colored path under $f_{1}$ with origin at $u_{3}$ (at $\left.v_{3}\right)$, then

(i) $u_{1}$ (respectively, $v_{1}$ ) is an endpoint of $Q^{\prime}$,

(ii) $Q^{\prime}$ passes through $u_{2}$ (through $v_{2}$ ), and

(iii) $Q^{\prime}$ does not pass through $v_{2}$ (through $u_{2}$ ).

Proof. We first prove (a). Let $Q$ be a maximal $(3,5)$-colored path with origin at $u_{3}$. Suppose that $u_{2}$ is not an endpoint of $Q$. Then, by making an interchange on $Q$, we obtain a proper coloring $f^{*}$ satisfying $f^{*}\left(u_{3}\right)=f^{*}\left(u_{2}\right)$. Thus we obtain a situation similar to the situation considered in Case B.2.2, which is impossible because in that case we can transform $f$ by interchanges to a required coloring $f^{\prime}$ satisfying (3.1). Hence $u_{2}$ is an endpoint of $Q$.

We now prove (b). Let $Q^{\prime}$ be a maximal path that is $(4,5)$-colored under $f_{1}$ and has origin at $u_{3}$. By interchanging colors on this path we obtain a coloring $f_{2}$.

Then $u_{1}$ is an endpoint of $Q^{\prime}$, because otherwise the coloring $f_{2}$ will satisfy $f_{2}\left(u_{3}\right)=f_{2}\left(u_{1}\right)$, which is impossible by the same reason as in the proof of the part (a) of this claim.

Suppose that $u_{2} \notin V\left(Q^{\prime}\right)$. Then by applying Lemma 3.2 to the path $v_{1} u_{1} u_{2} u_{3} u_{4}$, we deduce that one of conditions (II) and (III) of Lemma 3.2 holds. If the condition (III) holds, we may transform $f_{2}$ by interchanges on paths with colors from $\{3,4,5\}$ to a proper coloring $g$, such that $c \notin g\left(u_{i}\right) \cup g\left(u_{i+1}\right)$ for some color $c \in\{3,4,5\}$, and some $i \in\{1,2\}$. Then by recoloring $u_{i} u_{i+1}$ with $c$ we obtain a situation that is similar to the situation considered in Case A or Case B.1. If the condition (II) holds, then, by Lemma 3.2, we may transform $f_{2}$ by interchanges to another proper coloring and again obtain a situation which is similar to the situation considered in Case A.

Thus if $u_{2} \notin V\left(Q^{\prime}\right)$, then we obtain a situation which is similar to one of the situations considered in Case A and Case B.1, which is impossible because in those cases we can transform $f$ by interchanges to a required coloring $f^{\prime}$ satisfying (3.1). Therefore $u_{2} \in V\left(Q^{\prime}\right)$. 
Finally, $v_{2} \notin V\left(Q^{\prime}\right)$, because if $v_{2} \in V\left(Q^{\prime}\right)$, then $v_{1} v_{2} x_{1}$ is a maximal $(1,4)$-colored path under $f_{2}$, and by making an interchange on this path we obtain a coloring where only one edge adjacent to $u_{1} v_{1}$ is colored 1 , thus reducing this situation to the situation considered in Case A. But this is impossible because in that case we can transform $f$ by interchanges to a required coloring $f^{\prime}$ satisfying (3.1).

We continue the proof of the theorem. We have that the proper 5-edge coloring $f_{1}$ satisfies the conditions (3.5), (3.6), (3.7) and $P$ is either a cycle of length at least 8, or a path with $P=u_{l} u_{l-1} \ldots u_{2} u_{1} v_{1} v_{2} \ldots v_{k}$ where $l \geq 4, k \geq 4$ and $k+l \geq 9$. We assumed that it is impossible, using only interchanges, to transform $f$ (and therefore $f_{1}$ too) to a proper 5-edge coloring $f^{\prime}$ satisfying (3.1). Now we will show that for all possible $P$, despite our assumption, we can transform $f_{1}$ by interchanges either directly to a proper coloring $f^{\prime}$ satisfying (3.1), or to another proper coloring $\varphi$ where we have a situation which is similar to one of situations considered in Case A and Case B.1. This will lead to a contradiction, because in those cases we can transform $\varphi$, and therefore $f_{1}$ too, by a sequence of interchanges to a proper coloring $f^{\prime}$ satisfying (3.1).

Case B.2.3.1. $P$ is a path and only one of the vertices $u_{4}$ and $v_{4}$ is an endpoint of $P$.

Without loss of generality we assume that $u_{4}$ is an endpoint of $P$. If $f_{1}\left(v_{4}\right)=\{1,2,3,4\}$, then we recolor $v_{3} v_{4}$ with color 5 , and thereafter make an interchange on $u_{4} u_{3} u_{2} u_{1} v_{1} v_{2} v_{3}$ and denote the obtained coloring by $f_{2}$. Then $u_{3} u_{2} y_{1}$ is a maximal $(1,5)$-colored path under $f_{2}$. By interchanging colors on this path and then recoloring $u_{4} u_{3}$ with color 1 we obtain a coloring where $u_{4} u_{3}$ and $u_{1} v_{1}$ are correct but not $v_{3} v_{4}$. However, $v_{3} v_{4}$ is incident to only one edge colored 1 . We have thus obtained a situation which is is similar to one of the situations considered in Case A.

Suppose now that $f_{1}\left(v_{4}\right)=\{1,2,3,5\}$. Then we consider a maximal path $Q$ that is $(4,5)$ colored under $f_{1}$ and has origin at $v_{3}$. It follows from Claim 1 that $v_{1}$ is an endpoint of $Q$ and $u_{2} \notin V(Q)$. By interchanging colors on $Q$ we obtain a proper coloring that we denote by $f_{2}$. Then we recolor $v_{3} v_{4}$ with 4 , make an interchange on the maximal $(1,2)$-colored path $u_{4} u_{3} u_{2} u_{1} v_{1} v_{2} v_{3}$ and denote the obtained coloring by $f_{3}$. Then $u_{3} u_{2} y_{1}$ is a maximal $(1,5)$ colored path under $f_{3}$ in $G$. By interchanging colors on this path and then recoloring $u_{3} u_{4}$ with 1 , we obtain a coloring where $u_{4} u_{3}$ and $u_{1} v_{1}$ are correct but not $v_{3} v_{4}$. As before, $v_{3} v_{4}$ is incident to only one edge colored 1 . Hence, we obtain a situation which is similar to one of the situations considered in Case A.

Suppose now that $f_{1}\left(v_{4}\right)=\{1,2,4,5\}$. Then we consider a maximal $(3,5)$-colored path $Q$ with origin at $v_{3}$. By Claim 1, $v_{2}$ is an endpoint of $Q$. By interchanging colors on $Q$, we obtain a coloring that we denote by $f_{2}$. We now recolor $v_{3} v_{4}$ with 3 and then interchange colors on the maximal $(1,2)$-colored path $u_{4} u_{3} u_{2} u_{1} v_{1} v_{2} v_{3}$ and denote the obtained coloring by $f_{3}$. Then $u_{3} u_{2} y_{1}$ is a maximal $(1,5)$-colored path under $f_{3}$. By interchanging colors on this path and then recoloring $u_{3} u_{4}$ with color 1 we obtain a situation similar to the situation considered in Case A.

Case B.2.3.2. $P$ is a cycle of length at least 8 or a path where none of the vertices $u_{4}$ and $v_{4}$ is an endpoint of $P$. 
Since $\{1,2\} \subset f_{1}\left(v_{4}\right)$ and $\{1,2\} \subset f_{1}\left(u_{4}\right)$, the set $f_{1}\left(v_{4}\right)$ as well as the set $f_{1}\left(u_{4}\right)$ is one of the sets $\{1,2,3,4\},\{1,2,3,5\}$ and $\{1,2,4,5\}$. Furthermore, by symmetry in (3.5), we have to consider the following six cases only:

- $f_{1}\left(v_{4}\right)=f_{1}\left(u_{4}\right)=\{1,2,3,4\}$,

- $f_{1}\left(v_{4}\right)=f_{1}\left(u_{4}\right)=\{1,2,3,5\}$,

- $f_{1}\left(v_{4}\right)=f_{1}\left(u_{4}\right)=\{1,2,4,5\}$,

- $f_{1}\left(u_{4}\right)=\{1,2,3,4\}$ and $f_{1}\left(v_{4}\right)=\{1,2,3,5\}$,

- $f_{1}\left(u_{4}\right)=\{1,2,3,4\}$ and $f_{1}\left(v_{4}\right)=\{1,2,4,5\}$,

- $f_{1}\left(u_{4}\right)=\{1,2,3,5\}$ and $f_{1}\left(v_{4}\right)=\{1,2,4,5\}$.

$f_{1}\left(v_{4}\right)=f_{1}\left(u_{4}\right)=\{1,2,3,4\}$ : Recolor $v_{3} v_{4}$ and $u_{3} u_{4}$ with 5 and then make an interchange on the maximal $(1,2)$-colored path containing $u_{1} v_{1}$. Then $u_{4} u_{3} u_{2} y_{1}$ and $v_{4} v_{3} v_{2} x_{1}$ are maximal disjoint $(1,5)$-colored paths. By interchanging colors on these paths we obtain a proper coloring $f^{\prime}$ satisfying (3.1).

$f_{1}\left(u_{4}\right)=f_{1}\left(v_{4}\right)=\{1,2,3,5\}$ : Let $Q_{1}$ and $Q_{2}$ be maximal $(4,5)$-colored paths with origin at $u_{3}$ and $v_{3}$, respectively. Then by Claim 1, $u_{1}$ is an endpoint of $Q_{1}, v_{1}$ is an endpoint of $Q_{2}, u_{2} \notin V\left(Q_{2}\right)$ and $v_{2} \notin V\left(Q_{1}\right)$. Note also that this implies that $Q_{1}$ and $Q_{2}$ are disjoint. After interchanging colors on $Q_{1}$ and $Q_{2}$ we may recolor $u_{3} u_{4}$ and $v_{3} v_{4}$ with 4. Next, interchange colors on the maximal $(1,2)$-colored path $u_{3} u_{2} u_{1} v_{1} v_{2} v_{3}$. By Claim 1 , $u_{2} \in V\left(Q_{1}\right)$ and $v_{2} \in V\left(Q_{2}\right)$, which implies that both $u_{4} u_{3} u_{2} y_{1}$ and $v_{4} v_{3} v_{2} x_{1}$ are maximal $(1,4)$-colored paths, and by interchanging colors on these paths we obtain a proper coloring $f^{\prime}$ satisfying (3.1).

$f_{1}\left(u_{4}\right)=f_{1}\left(v_{4}\right)=\{1,2,4,5\}$ : We proceed similarly as in the preceding paragraph, but instead consider $(3,5)$-colored paths $Q_{1}$ and $Q_{2}$ with origin at $u_{3}$ and $v_{3}$, respectively. By Claim 1, $Q_{1}$ and $Q_{2}$ have endpoints $u_{2}$ and $v_{2}$, respectively. Note also that this implies that $Q_{1}$ and $Q_{2}$ are disjoint. Interchange colors on $Q_{1}$ and $Q_{2}$, and then recolor $u_{3} u_{4}$ and $v_{3} v_{4}$ with 3. Next, we make an interchange on the maximal $(1,2)$-colored path containing $u_{1} v_{1}$ and denote the obtained coloring by $f_{2}$. The paths $u_{4} u_{3} u_{2} y_{1}$ and $v_{4} v_{3} v_{2} x_{1}$ are maximal disjoint $(1,3)$-colored paths under $f_{2}$. Interchange colors on these paths to obtain a proper coloring $f^{\prime}$ satisfying (3.1).

$f_{1}\left(u_{4}\right)=\{1,2,3,4\}$ and $f_{1}\left(v_{4}\right)=\{1,2,3,5\}$ : Then we consider a maximal $(4,5)$-colored path $Q$ under $f_{1}$ with origin at $v_{3}$. By Claim 1, $v_{1}$ is an endpoint of $Q, v_{2} \in V(Q)$ and $u_{2} \notin V(Q)$. First by interchanging colors on $Q$, and thereafter recoloring $u_{3} u_{4}$ with 5 and $v_{3} v_{4}$ with 4 we obtain a coloring $f_{2}$. Next, we interchange colors on the maximal $(1,2)$-colored path $u_{3} u_{2} u_{1} v_{1} v_{2} v_{3}$ and denote the obtained coloring by $f_{3}$. Then $u_{4} u_{3} u_{2} y_{1}$ is a maximal $(5,1)$ colored path under $f_{3}$ and by interchanging colors on this path we obtain a proper coloring $f_{4}$ under which $u_{4} u_{3}$ and $u_{1} v_{1}$ are correct, but not $v_{3} v_{4}$. However, $v_{3} v_{4}$ is adjacent to only 
one edge colored 1, so we have obtained a situation which is similar to the one considered in Case A.

$f_{1}\left(u_{4}\right)=\{1,2,3,4\}$ and $f_{1}\left(v_{4}\right)=\{1,2,4,5\}$ : Then we consider a maximal path $Q$ that is $(3,5)$-colored under $f_{1}$ and has origin at $v_{3}$. By Claim 1, $v_{2}$ is an endpoint of $Q$. We now interchange colors on $Q$, then recolor $u_{3} u_{4}$ and $v_{3} v_{4}$ with 5 and 3 , respectively, and denote the obtained coloring by $f_{2}$. Thereafter make an interchange on the maximal $(1,2)$-colored path $u_{3} u_{2} u_{1} v_{1} v_{2} v_{3}$. Then $u_{4} u_{3} u_{2} y_{1}$ is a maximal $(5,1)$-colored path under the new coloring $f_{3}$. By interchanging colors on this path we obtain a proper coloring under which $u_{4} u_{3}$ and $u_{1} v_{1}$ are correct, but not $v_{3} v_{4}$. However, $v_{3} v_{4}$ is adjacent to only one edge colored 1 , so we have obtained a situation which is similar to the one considered in Case A.

$f_{1}\left(u_{4}\right)=\{1,2,3,5\}$ and $f_{1}\left(v_{4}\right)=\{1,2,4,5\}$ : Let $Q_{1}$ be a maximal $(4,5)$-colored path with origin at $u_{3}$. Then, by Claim 1, $u_{1}$ is an endpoint of this path, and $u_{2} \in V\left(Q_{1}\right)$ and $v_{2} \notin V\left(Q_{1}\right)$. Interchange colors on $Q_{1}$ and denote the obtained coloring by $f_{2}$. Next, we consider a maximal $(3,5)$-colored path $Q_{2}$ with origin at $v_{3}$. Similarly as in proof of Claim 1 , it can be shown that $v_{2}$ is an endpoint of $Q_{2}$. Interchange colors on $Q_{2}$ and denote the obtained coloring by $f_{3}$. We recolor $v_{3} v_{4}$ and $u_{3} u_{4}$ with colors 3 and 4 , respectively, and thereafter interchange colors on the maximal $(1,2)$-colored path $u_{3} u_{2} u_{1} v_{1} v_{2} v_{3}$. Denote this new coloring by $f_{4}$. Then $v_{4} v_{3} v_{2} x_{1}$ is a maximal $(1,3)$-colored path under $f_{4}$. By interchanging the colors on this path we obtain a situation which is similar to the situation considered in Case A.

We showed that for all possible $P$ we can transform $f_{1}$ by interchanges either directly to a proper coloring $f^{\prime}$ satisfying (3.1), or to another proper coloring $\varphi$ where we have a situation which is similar to one of situations considered in Case A and Case B.1. In both cases we can reach a proper coloring $f^{\prime}$ satisfying (3.1) by a sequence of interchanges. Since $f_{1}$ was obtained from $f$ also by a sequence of interchanges, we can obtain $f^{\prime}$ from $f$ by interchanges too. This lead to a contradiction with our assumption that it is impossible, using only interchanges, to transform $f$ to a proper 5-edge coloring $f^{\prime}$ satisfying (3.1).

Proof of Theorem 1.3. Let $G$ be a graph with $\chi^{\prime}(G)=\Delta(G)=4$. Furthermore, let $h$ be a proper 4-edge coloring of $G$ and $f$ be a proper $t$-edge coloring of $G$, where $t \geq 5$. We will show that $f$ can be transformed to $h$ by a sequence of interchanges.

If $t>5$, then by Vizing's algorithm $f$ can be transformed into a proper 5 -edge coloring using only interchanges (see e.g. [12]); the original problem now is reduced to the problem of transforming the obtained proper 5 -edge coloring to $h$. Thus we may assume that $t=5$.

Let $m$ denote the minimum degree of $G, 1 \leq m \leq 4$. We define graphs $G_{0}, G_{1}, \ldots, G_{4-m}$ and for each $G_{i}$ two proper colorings $f_{i}$ and $h_{i}$ in the following way:

1) $G_{0}=G$ and $f_{0}=f, h_{0}=h$.

2) Suppose that $G_{i}, f_{i}$ and $h_{i}$ are already defined for some $i \geq 0$. If $i=4-m$, then stop. Otherwise take two disjoint copies $G_{i}^{\prime}$ and $G_{i}^{\prime \prime}$ of $G_{i}$, with $V\left(G_{i}^{\prime}\right)=\left\{x^{\prime}: x \in V\left(G_{i}\right)\right\}$ and $V\left(G_{i}^{\prime \prime}\right)=\left\{x^{\prime \prime}: x \in V\left(G_{i}\right)\right\}$. Then we define $G_{i+1}$ as the graph obtained from $G_{i}^{\prime}$ and $G_{i}^{\prime \prime}$ by joining $x^{\prime}$ and $x^{\prime \prime}$ with an edge, for each vertex $x \in V\left(G_{i}\right)$ with degree less than 4 . The coloring $f_{i}$ of $G_{i}$ induces a proper 5 -edge coloring $f_{i+1}$ of $G_{i+1}$ in the following way: we color 
the copies $G_{i}^{\prime}$ and $G_{i}^{\prime \prime}$ in the same way as $G_{i}$, and then color the edge joining $x^{\prime}$ to $x^{\prime \prime}$ with a color from $\{1,2,3,4\}$ which is not used to color an edge incident with $x$ in $G_{i}$. Similarly, the coloring $h_{i}$ of $G_{i}$ induces a proper 4-edge coloring $h_{i+1}$ on $G_{i+1}$.

Clearly, the graph $G_{4-m}$ is a 4-regular graph. Furthermore, $h_{i}$ is a proper 4-edge coloring and $f_{i}$ is a proper 5 -edge coloring of $G_{i}$, for $i=0,1, \ldots, 4-m$. Then, by Theorem 3.4 , the proper 5-edge coloring $f_{4-m}$ of the graph $G_{4-m}$ can be transformed into the proper 4-edge coloring $h_{4-m}$ of $G_{4-m}$ by a sequence of interchanges. It is clear that these interchanges also define a sequence of proper colorings of $G$, beginning with $f$ and ending with $h$, such that each intermediate coloring in this sequence either is the same as the previous coloring or obtained from the previous coloring by either one interchange or a sequence of interchanges.

This completes the proof of Theorem 1.3.

\section{Proof of Theorem 1.6}

In this section we prove Theorem 1.6.

Proof of Theorem 1.6. Every graph $G$ with $\Delta(G) \geq 5$ where $G_{\geq 5}$ is acyclic is a Class 1 graph because the graph $G_{\Delta}$ is acyclic.

Let $\mathcal{A}$ denote the set of graphs $G$ with $\Delta(G) \geq 5$ where $G_{\geq 5}$ is acyclic and all Class 1 graphs with maximum degree 4 . We will show that for every $G \in \mathcal{A}$ all proper $(\Delta(G)+1)$ edge colorings are Kempe equivalent. The proof is by induction on the maximum degree $\Delta(G)$.

By Corollary 1.4, the proposition is true for all graphs in $\mathcal{A}$ with maximum degree 4 . Suppose that the proposition is true for all graphs in $\mathcal{A}$ with maximum degree less than $k$, $k \geq 5$, and let $G \in \mathcal{A}$ be a graph with $\Delta(G)=k$. Then $\chi^{\prime}(G)=k$. Consider a proper $k$-edge coloring $h$ of $G$. As in the proof of Theorem 1.1 we can assume that $M(h, k)$ is a maximal matching of $G$.

In order to show that any two proper $(k+1)$-edge colorings of $G$ are Kempe equivalent it is sufficient to show that any proper $(k+1)$-edge coloring of $G$ can be transformed to $h$ by a sequence of interchanges. Let $\varphi$ be an arbitrary proper $(k+1)$-edge coloring of $G$. By Proposition 2.1, $\varphi$ can be transformed by a sequence of interchanges to a proper $k$-edge coloring $\varphi_{1}$. Then the coloring $\varphi_{1}$ can be transformed to a proper $(k+1)$-edge coloring by sequentially recoloring the edges in the matching $M(h, k)$ with color $k+1$. Clearly, the obtained proper $(k+1)$-edge coloring $f$ satisfies the condition $M(h, k)=M(f, k+1)$. Furthermore, $f$ is obtained from $\varphi$ by a sequence of interchanges.

Consider the graph $G_{1}=G-M(h, k)$. The proper $k$-edge coloring $h$ of $G$ induces a proper $(k-1)$-edge coloring $h^{\prime}$ of $G_{1}$ and the proper $(k+1)$-edge coloring $f$ of $G$ induces a proper $k$-edge coloring $f^{\prime}$ of $G_{1}$. Clearly, $\chi^{\prime}\left(G_{1}\right)=k-1$. Therefore, $h^{\prime}$ is a proper $\chi^{\prime}\left(G_{1}\right)$-edge coloring and $f^{\prime}$ is a proper $\left(\chi^{\prime}\left(G_{1}\right)+1\right)$-edge coloring of $G_{1}$.

Moreover, $\Delta\left(G_{1}\right)=k-1$, because $G$ is a Class 1 graph and $k=\chi^{\prime}(G)$. Furthermore, $G_{1} \in \mathcal{A}$. Then, by the induction hypothesis, $f^{\prime}$ and $h^{\prime}$ are Kempe equivalent. This implies that the coloring $f$ of $G$ can be transformed to the coloring $h$ by a sequence of interchanges.

Thus for any graph $G \in \mathcal{A}$ with $\Delta(G)=k$ all proper $(k+1)$-edge colorings are Kempe equivalent. The proposition follows by the principle of induction. 


\section{References}

[1] A.S. Asratian, A note on transformations of edge colorings of bipartite graphs, Journal of Combinatorial Theory B 99 (2009), 814-818

[2] A. S. Asratian, A. Mirumian, Transformations of edge colorings of a bipartite multigraph and their applications, Soviet Math. Doklady 43 (1991), 1-3.

[3] C. Berge, J. C. Fournier, A short proof for a generalization of Vizing's theorem Journal of Graph Theory 15 (1991), 333-336

[4] J. C. Fournier, Coloration des aretes dun graphe, Cahiers du CERO (Bruxelles) 15 (1973) 311-314.

[5] T. R. Jensen, B. Toft, Graph Coloring problems, Wiley Interscience, 1995.

[6] J. McDonald, B. Mohar, D. Scheide, Kempe Equivalence of Edge-colorings in Subcubic and Subquartic Graphs, Journal of Graph theory 70 (2012), 226-239.

[7] B. Mohar, Kempe Equivalence of colorings, Graph Theory in Paris, Proceedings of a Conference in Memory of Claude Berge, Birkhäuser, Basel, 2006, 287-297.

[8] M. Stiebitz, B. Toft, D. Scheide, L.M. Favrholdt, Graph edge colouring: Vizing's theorem and Goldberg's conjecture, Wiley Interscience, 2012.

[9] V. G. Vizing, On an estimate of the chromatic class of a p-graph, Diskret analiz 3 (1964), 25-30.

[10] V. G. Vizing, The chromatic class of a multigraph (in Russian), Kibernetika (Kiev) 3 (1965), 29-39.

[11] V. G. Vizing, Some unsolved problems in graph theory (in Russian), Uspekhi Mat. Nauk 23 (1968), 117-134.

[12] D. B. West, Introduction to Graph Theory, 2nd ed, Prentice-Hall. 